\title{
Dynamics of a Stabilized Radion and Duality
}

\author{
Zackaria Chacko, ${ }^{a}$ Rashmish K. Mishra, ${ }^{a}$ and Daniel Stolarski ${ }^{a, b}$ \\ ${ }^{a}$ Maryland Center for Fundamental Physics, Department of Physics, \\ University of Maryland, College Park, MD 20742-4111 \\ ${ }^{b}$ Department of Physics and Astronomy, \\ Johns Hopkins University, Baltimore, MD 21218
}

\section{Abstract:}

We construct the effective theory of the graviscalar radion in the Randall-Sundrum scenario, taking into account effects arising from the stabilization of the extra dimension through the Goldberger-Wise mechanism. We explore the conditions under which the radion can remain light, and determine the corrections to its couplings to Standard Model (SM) states when the effects of stabilization are taken into account. We show that in the theories of interest for electroweak symmetry breaking that have a holographic dual, the presence of a light radion in the spectrum is not a robust prediction of the framework, but is in fact associated with mild tuning. We find that corrections to the form of the radion couplings to Standard Model particles arising from effects associated with brane stabilization are suppressed by the square of the ratio of the radion mass to the Kaluza-Klein scale. These corrections are small in theories where the radion is light, and are generally subleading, except in the case of couplings to the SM gluons and photon, when they can sometimes dominate. The AdS/CFT correspondence relates the radion in Randall-Sundrum models to the dilaton in theories where a strongly coupled conformal symmetry is spontaneously broken. We show that the discrepancies in the literature between the results for the dilaton and the radion can be traced to the omission of selfinteraction terms that would otherwise dominate the potential for the Goldberger-Wise scalar near the infrared brane. In the dual picture, this corresponds to neglecting the corrections to the scaling behavior of the operator that breaks conformal symmetry when it grows large. With the inclusion of these self-interaction terms, we find good agreement between the results on the two sides of the correspondence. 


\section{Contents}

1 Introduction 1

2 Stabilization of the Extra Dimension 3

3 Radion Couplings $\quad 13$

4 The Dual Picture 18

5 Conclusion $\quad 24$

A $5 D$ Gravity without a GW Scalar $\quad 25$

B Radion Mass $\quad 26$

C NDA Estimation of Parameters $\quad 29$

\section{Introduction}

For almost four decades the Standard Model (SM) has enjoyed remarkable success in describing the strong and electroweak interactions. The recent discovery of a new particle with mass close to $125 \mathrm{GeV}[1,2]$, if confirmed as the SM Higgs, will cement the SM as the correct effective description of nature at energies below the weak scale. However, there are good reasons to expect that the success of the SM will not continue. Within the SM there is no understanding of the stability of the weak scale under radiative corrections $[3,4]$ : the hierarchy problem. A solution to this problem requires new physics close to the weak scale that may be within reach of the Large Hadron Collider (LHC). Furthermore, the SM does not explain the hierarchy in the fermion masses or admit a dark matter candidate.

Among the many candidate theories that address the hierarchy problem, models based on the warped extra dimensional framework proposed by Randall and Sundrum [5] are among the most promising. Randall-Sundrum (RS) models that incorporate a custodial isospin symmetry $[6,7]$ can be consistent with precision electroweak measurements even if the Kaluza-Klein (KK) scale is as low as a few $\mathrm{TeV}$, allowing the hierarchy problem to be addressed. This has allowed calculable, holographic realizations of technicolor [8], and of the Higgs as a pseudo-Nambu-Goldstone boson (pNGB) [9, 10], to be constructed. The RS framework naturally accommodates new approaches to the flavor problem of the SM [11-13], and to the dark matter puzzle [14-16]. Apart from these attractive theoretical features, RS models have very distinctive collider signatures [17-19], which may help in distinguishing them from other theories. 
In RS models, fluctuations in the inter-brane separation are associated with a graviscalar field, the radion [5]. Several authors have studied the dynamics of the radion in this framework [20] and determined its couplings to the SM states, both in the case when the SM fields are localized to the infrared (IR) brane [21-24] and the case when they are in the bulk $[25,26]$. In the original RS construction, the radion couplings are fixed by $5 D$ diffeomorphism, but it is massless, corresponding to the fact that the distance between the two branes is a free parameter. Once the geometry is stabilized using, for example, the Goldberger-Wise (GW) mechanism [20], the inter-brane separation is fixed and the radion acquires a mass. In general, we expect corrections to the radion couplings arising from effects associated with the dynamics that stabilizes the geometry. These corrections, which may be significant, have not been taken into account in previous analyses.

The AdS/CFT correspondence [27-30], relates the original RS two brane model to strongly coupled large $N_{c}$ gauge theories that exhibit a conformal symmetry which is broken spontaneously [31, 32]. The brane localized SM Higgs of the RS model is identified with a composite of the strong dynamics on the four dimensional side of the correspondence $[9$, 10, 31]. The low energy spectrum of any theory where conformal symmetry is realized non-linearly contains a massless scalar, the dilaton [33-38], which can be thought of as the Nambu-Goldstone boson (NGB) associated with the breaking of scale invariance. In the correspondence, the massless radion of the RS model is identified with the dilaton [31, 32]. The mechanism that stabilizes the brane separation, and thereby generates a mass for the radion, corresponds to the presence of explicit conformal symmetry violating effects in the strongly coupled theory.

Several authors have studied the dynamics of the dilaton in the context of theories where strong conformal dynamics constitutes the ultraviolet (UV) completion of the physics that drives electroweak symmetry breaking [32, 39-46]. The mass of the dilaton [42-44, 46] and its couplings to SM states, incorporating conformal symmetry violating effects [43, 44], have been determined. However, at present there is only very imperfect agreement between the results in the literature for the dilaton mass on the CFT side of the correspondence and the radion mass in RS models. The reasons for this discrepancy, and its resolution, remain to be understood.

In this paper we construct the effective theory of the radion in RS models, taking particular care to include effects arising from the dynamics that stabilizes the geometry. We explore the conditions under which the radion can remain light and determine the form of its couplings to Standard Model (SM) states. We show that the mass of the radion can naturally lie below the Kaluza-Klein scale if all the parameters in the potential for the GW scalar lie below their natural strong coupling values. Alternatively, the spectrum of states will include a light radion if the tension of the IR brane is tuned to lie close to its value in the original RS model. The original RS setup where the radion is exactly massless may be thought of as an extreme case of this fine-tuning. Since neither of these conditions is in general expected to be satisfied in the theories of interest for electroweak symmetry breaking that have a holographic dual, the presence of a light radion in the spectrum is not a generic prediction of this framework, but is instead associated with mild tuning. We find that corrections to the form of the radion couplings to SM fields arising from effects 
associated with brane stabilization are suppressed by the square of the ratio of the radion mass to the Kaluza-Klein scale, and are small in theories where the radion is light. These corrections are generally subleading, except in the case of radion couplings to the SM gluons and photon localized on the IR brane, when they can sometimes dominate.

Holography decrees that the physics of the radion correspond to that of the dilaton in the dual description. We find good agreement between our results for the radion, and those in the literature for the dilaton. We are also able to arrive at a holographic interpretation of our results for the radion. As part of our analysis, we resolve the discrepancy in the literature between the results for the dilaton mass on the CFT side of the correspondence, and the radion mass in RS models. In theories where the dilaton mass lies below the scale of strong dynamics, the mass squared of the dilaton is known to depend on the scaling dimension of the operator $\mathcal{O}$ that leads to the breaking of conformal symmetry. Near the breaking scale where this operator is large, its scaling behavior differs significantly from that at the fixed point. We trace the reason for the discrepancy between the expressions for the radion mass on the two sides of the correspondence to the fact that this effect has not previously been systematically taken into account in RS models. We show that incorporating this effect corresponds to the addition of non-linear terms in the bulk potential for the GW scalar. Once this effect is taken into account, we obtain good agreement between the AdS and CFT sides of the correspondence.

The organization of rest of the paper is as follows. In section 2, we consider the effect of stabilization of the extra dimension by a GW scalar $\Phi$ and construct the low energy effective theory. In section 3 we determine the couplings of the radion to SM fields on the visible brane taking the effects of stabilization into account. In section 4 , we relate the obtained results to the dual picture making use of the AdS/CFT dictionary. We conclude in section 5. Various technical details are given in the appendices.

\section{Stabilization of the Extra Dimension}

In this section we construct the low energy effective theory of the radion incorporating the effects of the GW stabilization mechanism. Our primary objective will be to determine the dependence of the radion mass on the parameters of the extra dimensional theory. Following Goldberger and Wise we consider a scenario where a $5 D$ scalar field $\Phi$ acquires a vacuum expectation value (VEV) whose value depends on the location in the extra dimension [20]. However, we deviate from the original GW construction in that we allow the IR brane tension to be detuned away from its fine-tuned value in the original RS model, and also include non-linear self interaction terms for the GW field $\Phi$ in the bulk. As we shall see, this alters the parametric dependence of the radion mass. We will later elaborate on the significance of this by invoking the dual picture in section 4 . 


\subsection{Mass of the Radion}

We begin by considering the $5 D$ gravity action in the absence of any stabilization mechanism.

$$
\mathcal{S}_{G R}^{5 D}=\int d^{4} x \int_{-\pi}^{\pi} d \theta\left[\sqrt{G}\left(-2 M_{5}^{3} \mathcal{R}[G]-\Lambda_{b}\right)-\sqrt{-G_{h}} \delta(\theta) T_{h}-\sqrt{-G_{v}} \delta(\theta-\pi) T_{v}\right],
$$

where $M_{5}$ is the $5 D$ Planck mass, $\Lambda_{b}$ is the bulk cosmological constant and $T_{h}, T_{v}$ are the brane tensions at the hidden (UV) and visible (IR) brane respectively. We are using the $(+,-,-,-,-)$ sign convention for the $5 D$ metric. The classical solution for the geometry is a slice of $\mathrm{AdS}_{5}$ compactified on a circle $\mathcal{S}_{1}$ with $\mathcal{Z}_{2}$ orbifolding. The background metric $G_{M N}$ is then given as

$$
d s^{2}=e^{-2 k r_{c}|\theta|} \eta_{\mu \nu} d x^{\mu} d x^{\nu}-r_{c}^{2} d \theta^{2} \quad-\pi \leq \theta \leq \pi .
$$

To obtain a static solution of this form we have set $\Lambda_{b} / k=T_{v}=-T_{h}=-24 M_{5}^{3} k$, while $r_{c}$ is an arbitrary constant. To parametrize the light degrees of freedom, we promote $r_{c}$ and $\eta_{\mu \nu}$ to dynamical fields by letting $r_{c} \rightarrow r(x)$ and $\eta_{\mu \nu} \rightarrow g_{\mu \nu}(x)$, which are associated with the radion and the graviton fields respectively. After this replacement in the metric, we plug the metric back in the action and integrate over the extra dimension to obtain the effective theory for the $4 D$ graviton $g_{\mu \nu}(x)$ and the canonically normalized radion field $\varphi(x)$, see appendix A for details. Up to exponentially small corrections, this is given by

$$
\mathcal{S}_{G R}^{4 D}=\frac{2 M_{5}^{3}}{k} \int d^{4} x \sqrt{-g} \mathcal{R}\left[g_{\mu \nu}\right]+\int d^{4} x \sqrt{-g}\left(\frac{1}{2} \partial_{\mu} \varphi \partial^{\mu} \varphi-V(\varphi)\right) .
$$

From this we see that the $4 D$ Planck mass is given by $M_{4}^{2}=M_{5}^{3} / k$. The canonically normalized field $\varphi(x)$ is related to $r(x)$ as

$$
\varphi(x)=F e^{-k \pi r(x)}
$$

where $F$ is defined as $F=\sqrt{24 M_{5}^{3} / k}=\sqrt{24} M_{4}$. The potential generated for the radion field from the $5 D$ gravity sector is given by

$$
V_{G R}(\varphi)=\frac{\varphi^{4}}{F^{4}}\left(T_{v}-\frac{\Lambda_{b}}{k}\right)
$$

and vanishes identically for the tuned values of $\Lambda_{b}$ and $T_{v}$ in the original RS solution.

This naive procedure of promoting $\eta_{\mu \nu}$ and $r_{c}$ to dynamical fields leads to a parametrization of the light modes that does not solve the linearized Einstein equations [24, 47]. One may therefore worry that the low energy effective field theory (EFT) could get large corrections from the heavy fields. However, this turns out not to be a concern, as shown in [48]. A general parametrization of the fluctuations that includes both heavy and light modes can be written as

$d s^{2}=e^{-2 k r(x)|\theta|}\left[g_{\mu \nu}(x)+H_{\mu \nu}(x, \theta)\right] d x^{\mu} d x^{\nu}+2 H_{\theta \mu}(x, \theta) d x^{\mu} d \theta-r^{2}(x)\left[1+H_{\theta \theta}(x, \theta)\right] d \theta^{2}$. 
Here $g_{\mu \nu}(x)$ and $r(x)$ are again the light fields, while $H_{\mu \nu}(x, \theta), H_{\theta \mu}(x, \theta)$ and $H_{\theta \theta}(x, \theta)$ parameterize the heavy Kaluza-Klein (KK) excitations of the $5 D$ graviton after reducing to the four dimensional theory. This naive parametrization of the light degrees of freedom does not lead to large corrections from the heavy fields in the low energy effective theory as long there are no tadpole terms in the heavy fields with one derivative [48]. By $4 D$ Lorentz invariance, such a tadpole must multiply $H_{\mu \theta}$, but an explicit computation shows that $H_{\mu \theta}$ vanishes identically for the metric that solves the linearized Einstein equation [24, 47]. This ensures that our naive parametrization of light fields will indeed generate the correct low energy effective theory up to corrections that are suppressed by powers of the KK scale. This conclusion is not altered by the inclusion of the GW field.

In order to solve the hierarchy problem, the warp factor in the background geometry must be exponentially large. Defining $f=\langle\varphi\rangle=F e^{-k r_{c} \pi}$, we require a stable solution such that $f \ll F$. The radion potential in Eq. (2.5) generated from the gravitational part of the action does not allow for such a possibility dynamically, unless fine-tuned to vanish. The $\mathrm{VEV}$ of $\varphi$ either vanishes or is driven to infinity depending on the sign of the coefficient of the quartic. In the original RS solution to the hierarchy problem, this issue is addressed by setting the quartic coefficient to zero by tuning the tension of the visible brane such that $T_{v}=-24 M_{5}^{3} k=\Lambda_{b} / k$. The cosmological constant must also be tuned to zero by adjusting the tension of the hidden brane, $T_{h}=24 M_{5}^{3} k=-\Lambda_{b} / k$.

In a realistic RS scenario, a mechanism is needed to stabilize the geometry and give the radion a mass. Such a mechanism was proposed by Goldberger and Wise [20]. In this construction, a massive $5 D$ field $\Phi$ is sourced at the boundaries and acquires a VEV whose value depends on the location in the extra dimension. After integrating over the extra dimension, this generates a potential for the radion field in the low energy effective theory. In the original GW construction, the quartic potential for the radion in Eq. (2.5) was tuned to zero, and only the dynamics of the scalar field $\Phi$ contributed to the radion potential. Here we allow the quartic generated by the gravitational potential to be non-zero, and also include self-interaction terms in the potential for the GW scalar in the bulk. The effect of these additional terms, which are important in theories with a holographic dual, is to alter the parametric dependence of the radion mass.

We now construct the low energy effective theory for the radion that emerges after stabilization using the GW mechanism. The action for the GW scalar $\Phi$ is given by

$$
\mathcal{S}_{G W}=\int d^{4} x d \theta\left[\sqrt{G}\left(\frac{1}{2} G^{A B} \partial_{A} \Phi \partial_{B} \Phi-V_{b}(\Phi)\right)-\sum_{i=v, h} \delta\left(\theta-\theta_{i}\right) \sqrt{-G_{i}} V_{i}(\Phi)\right]
$$

Here $G_{h}$ and $G_{v}$ are the induced metrics at the brane locations, $\theta_{h}=0$ and $\theta_{v}=\pi$. The corresponding brane potentials are given by $V_{h}$ and $V_{v}$ respectively, while $V_{b}$ is the potential in the bulk. In our parametrization, the radion does not couple to the hidden brane at $\theta_{h}=0$. We therefore do not specify the form of $V_{h}$, but simply require that it fixes $\Phi$ at $\theta=0$ to be $k^{3 / 2} v$ and does not contribute to the hidden brane tension. Such a requirement can be easily arranged, and is in fact the one used in the original GW proposal. 
On the visible brane, we consider a simple potential for $\Phi$ of the form

$$
V_{v}(\Phi)=2 k^{5 / 2} \alpha \Phi
$$

where $\alpha$ is a dimensionless number. The choice of a linear potential for $\Phi$ on the IR brane is not special and is expected to present if $\Phi$ is not charged under any symmetries. Considering a more general brane potential does not alter our final conclusions.

The potential for the GW scalar $\Phi$ in the bulk has the general form

$$
V_{b}(\Phi)=\frac{1}{2} m^{2} \Phi^{2}+\frac{1}{3 !} \eta \Phi^{3}+\frac{1}{4 !} \zeta \Phi^{4}+\ldots
$$

Since we are in AdS space, the mass squared parameter $m^{2}$ for the GW scalar $\Phi$ can be negative without giving rise to instabilities as long as the condition $m^{2} / k^{2}+4>0$ is satisfied [49]. The theories of interest for electroweak symmetry breaking are characterized by a large hierarchy between the scales associated with the UV brane and the IR brane, $\exp \left(-k \pi r_{c}\right) \ll 1$. The size of the hierarchy is determined by the potential for the GW scalar, which fixes the brane spacing $r_{c}$. A large hierarchy can be obtained if the mass term in the bulk potential for the GW field is small in units of the inverse curvature $k$. As first observed by Goldberger and Wise [20], a value of $\left|m^{2} / k^{2}\right|$ of order a tenth suffices to generate the hierarchy between the Planck and weak scales.

In the class of RS models that possess a holographic dual, the parameters in the $5 D$ gravity theory and in the potential for the GW scalar are related to parameters in the dual CFT. The AdS/CFT correspondence associates the GW field $\Phi$ with a scalar operator $\mathcal{O}$ that deforms the dual CFT. Our primary focus is on the duals of $4 D$ theories where a nearly marginal deformation $\mathcal{O}$, although small in the UV, grows large in the IR, resulting in the breaking of the conformal symmetry. This dictates our choice of the values of the parameters in the $5 D$ theory. The mass of $\Phi$ is related to the scaling dimension of $\mathcal{O}$ at the fixed point, and $\left|m^{2} / k^{2}\right| \ll 1$ corresponds to the operator $\mathcal{O}$ being close to marginal. We choose $m^{2}<0$ to obtain a solution for $\Phi$ which grows in the IR. The dimensionless parameter $v$, which determines the value of $\Phi$ at the UV brane is also chosen to be small, allowing us to work to linear order in $v$. This corresponds to the operator $\mathcal{O}$ being a small deformation in the UV. The other parameters are taken to be of order their natural strong coupling values (see appendix $\mathrm{C}$ for a description of how to estimate the natural values of parameters), but with an important caveat. In our analysis, we neglect the back-reaction of the scalar field dynamics on the metric. In order for this approximation to be selfconsistent, the contribution of the GW field to the total energy density at any point must be smaller, by a factor of at least a few, than that of the cosmological constant. For this to be the case the detuning of the IR brane tension away from that in the original RS model must be smaller, by a factor of order a few, than its natural strong coupling value. The value of the parameter $\alpha$, which controls the value of $\Phi$ near the IR brane, must also be taken to be smaller than its natural value by a similar factor. We do not expect our general conclusions to be affected by the fact that we are working in a limit where the back-reaction is small. 
Given the action, we can solve for the classical field configuration $\Phi(\theta)$ in the RS background. The equation satisfied by $\Phi$ in the bulk with the boundary conditions resulting from brane potentials is given by

$$
\begin{array}{lcc}
\partial_{\theta}^{2} \Phi-4 k r_{c} \partial_{\theta} \Phi-r_{c}^{2} V_{b}^{\prime}(\Phi)=0 & \\
\theta=0 \quad: & \Phi=k^{3 / 2} v \\
\theta=\pi & : & \partial_{\theta} \Phi=-\alpha k^{3 / 2} k r_{c} .
\end{array}
$$

The classical equation is a second order differential equation and is not simple to solve analytically for a general bulk potential. If, however, $4 k r_{c} \gg 1$ and certain conditions are satisfied by the parameters in the bulk potential, then an approximate analytic solution may be obtained [42] using the methods of singular perturbation theory [50]. The key observation is that in the limit $4 k r_{c} \gg 1$, the bulk equation of motion in Eq. (2.10) possesses solutions in different regions of $\theta$ that have the property that one of the terms in Eq. (2.10) is small compared to the other two. In particular, there are self-consistent solutions for $\Phi$ in the bulk that satisfy the boundary condition on the UV brane such that the $\partial_{\theta} \Phi$ term and $V_{b}^{\prime}$ term are parametrically larger than the $\partial_{\theta}^{2} \Phi$ term. Within this approximation, the equation effectively becomes first order and is readily solved. The full solution to the equation displays boundary layer formation very near the $\theta=\pi$ boundary where the $\partial_{\theta}^{2} \Phi$ term cannot be self-consistently dropped. However, in this regime it is self-consistent to drop the potential term in favor of the $\partial_{\theta} \Phi$ and $\partial_{\theta}^{2} \Phi$ terms.

It follows that two independent approximate solutions to the second order differential equation can be obtained in the following way: once by balancing the $\partial_{\theta} \Phi$ term against the potential term, and once by balancing the $\partial_{\theta} \Phi$ term against the $\partial_{\theta}^{2} \Phi$ term. We will refer to these two equations (solutions) as the outer region (OR) and boundary region (BR) equations (solutions) respectively. The OR solution holds in the bulk, while the BR solution holds close to $\theta=\pi$ where a boundary layer is formed, as the name suggests. The thickness of the boundary layer is of the order $\sim 1 / 4 k r_{c}$. More specifically, the equations and their domains of validity ${ }^{1}$ are,

$$
\begin{aligned}
\text { OR : } & \frac{d \Phi}{d \theta}=-\frac{r_{c}}{4 k} V_{b}^{\prime}(\Phi) & & \left(0 \leq \theta \lesssim \pi-\frac{1}{4 k r_{c}}\right) \\
\text { BR : } & \frac{d^{2} \Phi}{d \theta^{2}}=4 k r_{c} \frac{d \Phi}{d \theta} & & \left(\pi-\frac{1}{4 k r_{c}} \lesssim \theta \leq \pi\right) .
\end{aligned}
$$

Notice that the BR solution is independent of the choice of potential $V_{b}$ and can be readily solved. After applying the boundary condition at $\theta=\pi$, it is given by

$$
\Phi_{B R}(\theta)=-\frac{k^{3 / 2} \alpha}{4} e^{4 k r_{c}(\theta-\pi)}+C .
$$

The yet unspecified constant $C$ is determined by requiring the BR solution to be consistent with the OR solution, using asymptotic matching to the OR solution [50]. This allows us

\footnotetext{
${ }^{1}$ For the remainder of this work, we give solutions for $0 \leq \theta \leq \pi$. Because of the $\mathcal{Z}_{2}$ orbifold symmetry, this is sufficient to reconstruct the solution for the entire space.
} 
to construct a smooth solution that is a good approximation to each of the two solutions in the appropriate region. It is clear that the BR solution is exponentially suppressed in the region $0 \leq \theta \lesssim \pi-1 / 4 k r_{c}$, but becomes important in the region $\pi-1 / 4 k r_{c} \lesssim \theta \leq \pi$, thereby justifying the approximations made. Different choices of $V_{b}$ change the OR solution, and therefore change the constant $C$ in the BR solution. The complete solution for $\Phi$ exhibits the universal feature of boundary layer formation close to $\theta=\pi$. As we shall see, this general characteristic is to be expected from the point of view of the holographic dual theory.

The potential for the GW scalar $\Phi$ in the bulk takes the form

$$
V_{b}(\Phi)=\frac{1}{2} m^{2} \Phi^{2}+\frac{1}{3 !} \eta \Phi^{3}+\frac{1}{4 !} \zeta \Phi^{4}+\ldots .
$$

We can then write the OR equation in a holographically suggestive form

$$
\frac{d \log \Phi}{d\left(k r_{c} \theta\right)}=-\frac{m^{2}}{4 k^{2}}-\frac{\eta}{8 \sqrt{k}} \frac{\Phi}{k^{3 / 2}}-\frac{\zeta k}{24} \frac{\Phi^{2}}{k^{3}}+\ldots .
$$

In what follows we restrict ourselves to specific forms of the bulk potential for the GW field. In particular, we will first study the case when $V_{b}(\Phi)$ consists of just a mass term, and then consider the case of a cubic self-interaction term. For each theory, we will describe the conditions on the parameters of the bulk potential for our approximation to be valid. We will then obtain the OR solution and study the resulting radion potential. These special cases will suffice to determine the general features of the solution.

\section{Massive GW Scalar with no Bulk Interactions}

We begin by considering the case when the potential for $\Phi$ is dominated by the mass term, and the higher powers of $\Phi$ can be neglected,

$$
V_{b}(\Phi)=\frac{1}{2} m^{2} \Phi^{2}
$$

The large hierarchy between the Planck and weak scales implies that $e^{-k r_{c} \pi}$ is a very small number. To obtain this hierarchy we require the bulk mass squared being small in units of the inverse curvature, namely $\epsilon=m^{2} / 4 k^{2}$ is a small number, $|\epsilon| \ll 1$. We also take $e^{-\epsilon k r_{c} \pi}$ to be an $\mathcal{O}(1)$ number. These conditions are also sufficient for our boundary layer analysis to be valid. The OR equation in this case takes the form

$$
\frac{d \log \Phi}{d\left(k r_{c} \theta\right)}=-\frac{m^{2}}{4 k^{2}}=-\epsilon .
$$

Applying the boundary condition at $\theta=0$, the solution is

$$
\Phi_{O R}(\theta)=k^{3 / 2} v e^{-\epsilon k r_{c} \theta} .
$$

Given this $\mathrm{OR}$ solution, the constant $C$ in the BR solution is fixed uniquely by requiring asymptotic matching to the OR solution, resulting in

$$
\Phi_{B R}(\theta)=-\frac{k^{3 / 2} \alpha}{4} e^{4 k r_{c}(\theta-\pi)}+k^{3 / 2} v e^{-\epsilon k r_{c} \pi} .
$$


Using the form of the OR and BR solutions, a smooth approximate solution for $\Phi$ that is valid in the entire region $0 \leq \theta \leq \pi$ and that matches on to both $\Phi_{B R}$ and $\Phi_{O R}$ is given by

$$
\Phi_{\text {approx }}(\theta)=-\frac{k^{3 / 2} \alpha}{4} e^{4 k r_{c}(\theta-\pi)}+k^{3 / 2} v e^{-\epsilon k r_{c} \theta} .
$$

For this choice of the bulk potential, an exact expression for $\Phi$ can be obtained (see appendix B), which matches very well with the approximate solution. Figure 1 indicates the matching of the BR and IR solutions to the approximate solution (1a), and shows the agreement of the approximate solution with the exact solution (1b).
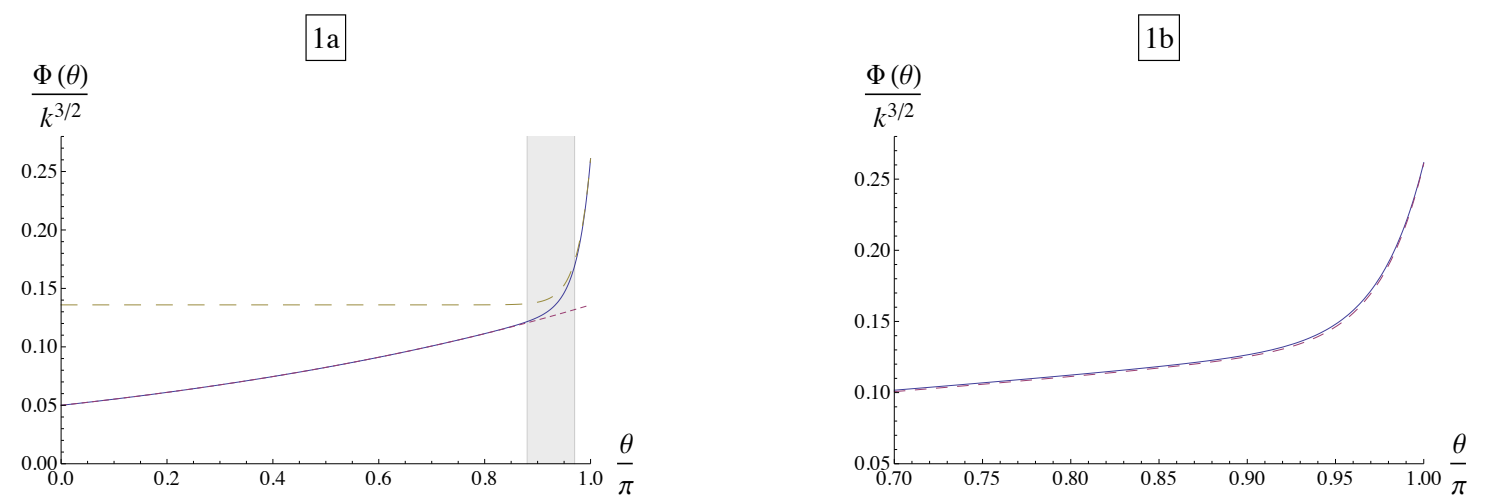

Figure 1. 1a: The approximate solution (solid line) matches well with the BR solution (large dotted) and the OR solution (small dotted) for $\epsilon=-0.1, k \pi r_{c}=10$. We have also taken $v=0.05$ and $\alpha=-0.5$. The shaded region separates the boundary region on its right from the outer region on its left. Asymptotic matching is done in the shaded region. 1b: The approximate solution (dotted) agrees well with the exact solution (solid) for the same parameter values, and we show the agreement near the $\theta=\pi$ boundary.

To calculate the resulting contribution to the radion potential, we promote $r_{c}$ to a dynamical field and insert the complete solution for $\Phi(\theta)$ with this replacement back into the action. Integrating over the extra dimension then generates the contribution to the radion potential (see appendix $\mathrm{B}$ for details). To leading order in $v$ and $\epsilon$, the resulting potential takes the form

$$
V_{G W}(\varphi)=k^{4}\left(\frac{\varphi}{F}\right)^{4}\left[2 \alpha v\left(\frac{\varphi}{F}\right)^{\epsilon}-\frac{\alpha^{2}}{4}\right] .
$$

Including the contribution from the gravity part of the action in Eq. (2.5), the full potential for the radion is given to leading order in $v$ and $\epsilon$ as

$$
\begin{aligned}
V(\varphi) & =V_{G R}(\varphi)+V_{G W}(\varphi) \\
& =2 k^{4} \alpha v\left(\frac{\varphi}{F}\right)^{4+\epsilon}+\left(T_{v}-\frac{\Lambda_{b}}{k}-\frac{k^{4} \alpha^{2}}{4}\right)\left(\frac{\varphi}{F}\right)^{4} .
\end{aligned}
$$

We can define $\tau=\left(T_{v}-\Lambda_{b} / k-k^{4} \alpha^{2} / 4\right) / k^{4}$ which represents the detuning of the brane tension away from the original RS solution. The first two terms in $\tau$ are purely gravitational, while the last term comes from the dynamics of the GW field on the visible brane 
and receives corrections higher order in $\epsilon$. With this, the minimum of the potential in Eq. (2.21) occurs at $\langle\varphi\rangle=f$ where the condition

$$
\left[\frac{f}{F}\right]^{\epsilon} \equiv e^{-\epsilon k r_{c} \pi}=-\frac{\tau}{2 \alpha v}+\mathcal{O}(\epsilon)
$$

is satisfied. For small $\epsilon$, an exponential hierarchy can be established between $f$ and $F$ provided the parameter $v$ which controls the value of $\Phi$ at the UV brane is also small. In particular, if $\epsilon$ is of order a tenth and the other parameters are of order their natural strong coupling values, then $v$ of order a tenth suffices to generate the hierarchy between the Planck and weak scales.

After $\varphi$ acquires a VEV, we parametrize the physical radion $\widetilde{\varphi}$ that corresponds to the fluctuations over the minimum as $\varphi=f \exp [\widetilde{\varphi} / f] \approx f+\widetilde{\varphi}$. The mass of the radion $\widetilde{\varphi}$ is given to leading order in $\epsilon$ as

$$
m_{\varphi}^{2}=-\frac{\epsilon \tau}{6} \frac{k^{3}}{M_{5}^{3}}\left(k e^{-k r_{c} \pi}\right)^{2} .
$$

The warped down curvature scale $k e^{-k r_{c} \pi} \equiv \widetilde{k}$ appears naturally in this expression, and is identified with the KK scale $m_{K K}$ in the $4 D$ theory. Including higher order terms in $V(\varphi)$ does not alter the results. At the minimum, the value of $T_{h}$ is tuned to set the $4 D$ cosmological constant to zero. Therefore, in the presence of a GW mechanism, only one fine-tuning is needed, which is the usual tuning to solve the cosmological constant problem.

We see from this analysis that $m_{\varphi}^{2} \sim|\epsilon|$, which is consistent with previous studies [51, 52 . Either sign of $\epsilon$ can give rise to a positive mass squared for the radion. In the case of $\epsilon>0$ we require $\tau<0$ and $\alpha v>0$, while for $\epsilon<0$ these conditions are reversed. (The condition on the sign of $\alpha v$ arises from requiring the consistency of the minimization condition.) From the holographic perspective, the case $\epsilon<0$ when the solution for $\Phi$ grows in the IR is particularly interesting, and is our primary focus. The parametric dependence of the dilaton mass on $\epsilon$ is robust and independent of the form of the potential for $\Phi$ on the visible brane. Since $\epsilon$ is small this would seem to suggest that the presence of a light dilaton is natural in this class of theories. However, this result assumed that the cubic self-interaction terms in the bulk potential for $\Phi$ could be neglected in favor of the mass terms. For this assumption to be self-consistent we require that the condition

$$
\frac{1}{3 !} \eta \Phi^{3} \ll \frac{1}{2} m^{2} \Phi^{2}
$$

be satisfied at all points in the bulk. Using the solution for $\Phi$ and noting that it has the largest magnitude at $\theta=\pi$, this translates to

$$
\left|\left(\frac{\eta}{8 \sqrt{k}}\right)\left(\tau+\frac{\alpha^{2}}{2}\right)\right| \ll 3|\epsilon \alpha| .
$$

For natural values of the parameters $\alpha, \tau$, and $\eta$, this condition is not expected to be satisfied. Therefore, in determining whether a light radion is present in the spectrum, it is in general necessary to consider the bulk self-interaction terms. 
However, there does exist a class of theories in which the condition Eq. (2.25) can naturally be satisfied. These are theories where the GW scalar is the pNGB of an approximate global symmetry that is spontaneously broken. Our results show that in such theories the radion can naturally be light, its mass scaling as $\sqrt{\epsilon}$, in the limit that back-reaction is small. Although in our analysis we only worked to linear order in the small parameter $v$, we have explicitly verified that this conclusion is not altered when higher powers of $v$ are included.

It is interesting to ask whether the conclusion that the radion is light continues to hold when the detuning of the IR brane tension away from the RS value is large, so that back-reaction cannot be neglected. Although our analysis, strictly speaking, is not valid in this regime, the fact that the radion mass continues to scale as $\sqrt{\epsilon}$ even as the detuning is taken towards its natural value suggests that this result does continue to hold. Although this is not conclusive, evidence from the CFT side of the correspondence also indicates that this is the case [43].

\section{Massive GW Scalar with a Cubic Interaction in Bulk}

Next we consider the effect of including self-interaction terms in the bulk for $\Phi$. In general, the bulk potential is expected to include all powers of $\Phi$, as in Eq. (2.13). However, we limit our analysis to the case of a cubic self-interaction term,

$$
V_{b}(\Phi)=\frac{1}{2} m^{2} \Phi^{2}+\frac{1}{3 !} \eta \Phi^{3} .
$$

This restriction is not expected to affect our conclusions because, in the limit that the parameters are such that the back-reaction is small, the trilinear term is expected to dominate. The reason is that small back-reaction implies that the VEV of $\Phi$ is small in units of AdS curvature, so even though all the coefficients of the interaction terms are of order their strong coupling values, the potential will be dominated by the interaction involving the lowest power of $\Phi$, the trilinear term.

With the bulk potential given by Eq. (2.26), the OR equation now becomes

$$
\frac{d \log \Phi}{d\left(k r_{c} \theta\right)}=-\epsilon-\frac{\eta}{8 \sqrt{k}}\left(\frac{\Phi}{k^{3 / 2}}\right) \text {. }
$$

We focus on the case when $\eta / 8 \sqrt{k}$ is close to its natural strong coupling value. We further work in the limit that $(\eta / 8 \sqrt{k})\left(\Phi / k^{3 / 2}\right)$ is large enough that $\epsilon$ can be neglected in Eq. (2.27). Although this latter condition is not necessarily satisfied at all points in the bulk, it is expected to be satisfied in the neighborhood of the IR brane. The reason is that in the theories of interest, while $\epsilon$ is required to be small in order to generate a large hierarchy, there is no corresponding condition on the cubic self-coupling. Since the radion wave function is localized near the IR brane, neglecting $\epsilon$ does not significantly affect the radion dynamics.

In the limit where the cubic dominates the potential, the OR solution is given by

$$
\Phi_{O R}(\theta)=\frac{k^{3 / 2} v}{1+\xi k r_{c} \theta} .
$$


Here $\xi=\eta v / 8 \sqrt{k}$ and we have imposed the boundary condition $\Phi(0)=k^{3 / 2} v$. In order for our boundary layer analysis to be self-consistent, we require that both $\alpha$ and $v$ to lie below their natural values by a factor that could be as small as a few. However, we have already chosen $v$ to be small in order to generate a large hierarchy. We have also set $\alpha$ somewhat smaller than its natural value so that gravitational back-reaction can be neglected. Hence our boundary layer analysis is valid without additional restrictions. Combining the OR and the BR solutions, we obtain the complete solution for $\Phi$ as

$$
\Phi(\theta)=-\frac{k^{3 / 2} \alpha}{4} e^{4 k r_{c}(\theta-\pi)}+\frac{k^{3 / 2} v}{1+\xi k r_{c} \theta} .
$$

We can now compute the potential for the radion to leading order in $v$ :

$$
V(\varphi)=k^{4}\left(\frac{\varphi}{F}\right)^{4}\left[\tau+\frac{1}{1-\xi \log (\varphi / F)}\left(2 \alpha v+\frac{\alpha^{2} \xi}{8}\right)\right] .
$$

We can define $w=2 \alpha v+\alpha^{2} \xi / 8$ and note that $w$ is small because it is linear in $v$. The potential is minimized when the condition

$$
\tau+\frac{w}{1-\xi \log (f / F)}=0
$$

is satisfied. We can solve for $\langle\varphi\rangle=f$,

$$
\left[\frac{f}{F}\right]^{\xi} \equiv e^{-\xi k r_{c} \pi}=e^{1+w / \tau}+\mathcal{O}(\xi) .
$$

This is more transparent when written in terms of the radius of the extra dimension,

$$
k \pi r_{c} \approx-\frac{w+\tau}{\xi \tau} \approx-\frac{1}{\xi} .
$$

To obtain a large warp factor $k \pi r_{c}$ must be larger than 1 . This condition is satisfied if $v$ is of order $10^{-2}$, which justifies the last approximation in Eq. (2.33). From Eqs. (2.30) and (2.31), we can compute the mass squared of the radion

$$
m_{\varphi}^{2}=\frac{\tau^{2} \xi}{6 w} \frac{k^{3}}{M_{5}^{3}}\left(k e^{-k r_{c} \pi}\right)^{2} .
$$

Details of the calculation are given in appendix B.

Putting in the natural values of the parameters given in appendix $\mathrm{C}$, we find that the radion mass is parametrically of order the KK scale, so that it is no longer light. Although our analysis neglected back-reaction, we do not expect that including this effect would alter our conclusions. If $\eta$, (as well as $\epsilon$ ), was tuned to be small, then Eq. (2.34) would predict a light radion. In that case, however, the quartic and higher order terms in the $\Phi$ bulk potential would give large contributions to the radion mass. If, however, all the terms in the $\Phi$ bulk potential are small in units of curvature, the radion will be light. This could be realized naturally, if, for example, the GW field corresponds to a pseudo-Goldstone boson.

We also see from Eq. (2.34) that the mass of the radion scales as $\tau$. Therefore a light radion can arise if $\tau$ lies below its natural strong coupling value. Small values of $\tau$ are associated with tuning, since this condition is not expected to be satisfied in general. However, the tuning of the radion mass is mild, scaling as $\tau$. Therefore a radion that lies a factor of 5 below the KK scale is only tuned at the level of 1 in $5(20 \%)$. 


\subsection{Mixing with Other Scalars}

Along with the radion, the other scalars in the low energy spectrum are the first few KK modes $\left\{\phi_{n}\right\}$ of the scalar $\Phi$ which in general mix with the physical radion $\widetilde{\varphi}$. Expanding the $\Phi$ action to linear order in $\widetilde{\varphi}$ and $\phi_{n}$, all terms without a spacetime derivative vanish because of the classical $\Phi$ equations of motion [32]. This implies there is only kinetic mixing between $\widetilde{\varphi}$ and $\phi_{n}$. The mixing coefficient $\kappa_{n}$ affects the physical radion and KK modes as

$$
\widetilde{\varphi} \rightarrow \widetilde{\varphi}-\kappa_{n} \frac{m_{n}^{2}}{m_{n}^{2}-m_{\varphi}^{2}} \phi_{n} \quad \phi_{n} \rightarrow \phi_{n}-\kappa_{n} \frac{m_{\varphi}^{2}}{m_{\varphi}^{2}-m_{n}^{2}} \widetilde{\varphi},
$$

where $m_{n}$ is the mass of the KK mode and $\widetilde{\varphi}$ is the radion field. We find that $\kappa_{n} \sim m_{\varphi}^{2} / m_{n}^{2}$ for both types of bulk $\Phi$ potentials considered in this section.

\section{Radion Couplings}

In this section we calculate the corrections to the radion couplings to SM fields that arise as a consequence of the GW stabilization mechanism. In the absence of the GW scalar, the radion being a massless scalar gravitational mode couples to the trace of the $5 D$ energy momentum tensor [22]. Its couplings are therefore completely fixed. This has been used to determine the couplings of the radion, both in the case when the SM fields are confined to the IR brane [20-24] and the case when they reside in the bulk of the space [25, 26]. In this paper we will consider SM fields confined to a brane, and leave the case of bulk fields for future work.

Once the GW scalar $\Phi$ is added to the theory, in general we expect interactions that couple $\Phi$ to the SM fields. Since the VEV of $\Phi$ depends on the brane spacing, and therefore on the background radion field, this effect contributes to the coupling of radion to SM fields. To understand this in detail, consider an arbitrary term in the Lagrangian that depends only on the SM fields,

$$
\mathcal{L} \supseteq \sqrt{G} f\left(\Psi_{i}, \mathcal{A}_{i}^{\mu}, \mathcal{H}_{i}\right)
$$

Here $f\left(\Psi_{i}, \mathcal{A}_{i}^{\mu}, \mathcal{H}_{i}\right)$ is a function of the SM fermions $\Psi_{i}$, the SM gauge fields $\mathcal{A}_{i}^{\mu}$ and the Higgs field $\mathcal{H}$. Then, one can also write the following interaction term in the Lagrangian involving the GW field $\Phi$,

$$
\mathcal{L} \supseteq \alpha_{\text {int }} \sqrt{G} f\left(\Psi_{i}, \mathcal{A}_{i}^{\mu}, \mathcal{H}_{i}\right) k^{-3 / 2} \Phi(x, \theta),
$$

where $\alpha_{\text {int }}$ is a dimensionless coupling constant. Terms containing higher powers of $\Phi$ in the interaction do not change our conclusions as we work to leading order in $v$. In theories with a holographic dual, we expect $\alpha_{\text {int }}$ to be of order its natural strong coupling value (see appendix $\mathrm{C}$ ).

In order to determine the radion couplings, we allow the GW field to fluctuate and KK expand the fluctuations over the classical value. This amounts to replacing

$$
\Phi(\theta) \rightarrow \Phi(\theta)+\sum_{n} f_{n}(\theta) \phi_{n}(x)
$$


in Eq. (3.2), where $\phi_{n}$ are the $\mathrm{KK}$ modes of the GW field $\Phi$. The radion couplings to the light fields contained in $f\left(\Psi_{i}, \mathcal{A}_{i}^{\mu}, \mathcal{H}_{i}\right)$ get contributions from two sources. Firstly, in the parametrization that we consider, the background value $\Phi(\theta)$ is a function of the background radion field $\varphi(x)$ after $r_{c}$ is made dynamical. Expanding $\Phi(\theta)$ about the radion VEV to linear order generates coupling to the physical radion $\widetilde{\varphi} \approx \varphi-f$ as

$$
\Phi(\theta) \rightarrow \Phi(\varphi, \theta)=\Phi(f, \theta)+\widetilde{\varphi} \partial_{\varphi} \Phi(f, \theta)+\ldots
$$

Secondly, the KK modes $\phi_{n}$ in general have a kinetic mixing with the radion, and thereby generate a radion coupling to the SM fields. From section 2.2, it follows that this effect scales as $m_{\varphi}^{4} / m_{K K}^{4}$ for a light radion. As we will see below, this effect is subleading and can be neglected.

We can schematically understand how the corrections to the radion couplings scale. For simplicity, we focus on the case where the SM fields live on the visible brane. When the bulk potential for $\Phi$ is dominated by the mass term, we find that the leading source of modification to the radion coupling, using Eqs. (3.4) and (2.19), scales as $\left.\partial_{\varphi} \Phi\right|_{\theta=\pi} \sim$ $\epsilon v e^{-\epsilon k r_{c} \pi}$. From the minimization condition Eq. (2.22) and the expression for the mass of the radion Eq. (2.23), we obtain

$$
\epsilon v e^{-\epsilon k r_{c} \pi} \sim \epsilon \tau \sim \frac{m_{\varphi}^{2}}{m_{K K}^{2}}
$$

We see that the correction scales as $\sim m_{\varphi}^{2} / m_{K K}^{2}$ and is small for a light radion.

In the case when the the bulk potential for $\Phi$ is dominated by the cubic self interaction term, using Eqs. (3.4) and (2.29), we find that the leading source of corrections to the radion coupling now scales as

$$
\left.\partial_{\varphi} \Phi\right|_{\theta=\pi} \sim v \xi /(1-\xi \log f / F)^{2} .
$$

From the expressions for the minimization condition Eq. (2.31) and the mass of the radion Eq. (2.34), we find that

$$
\frac{v \xi}{(1-\xi \log (f / F))^{2}} \sim \tau^{2} \sim \frac{m_{\varphi}^{2}}{m_{K K}^{2}},
$$

so that the corrections again scale as $\sim m_{\varphi}^{2} / m_{K K}^{2}$. It follows from this analysis that the form and magnitude of the leading corrections to the radion couplings does not depend on the details of the GW mechanism that generates the radion mass.

We now calculate the corrections to the radion couplings to SM fields in detail, focusing on the radion couplings to massive and massless gauge bosons, and to fermions. We will consider the case where the Higgs is the pNGB of an approximate global symmetry [5357]. In this scenario, mixing between the Higgs and the radion, which can otherwise be significant, is suppressed [23], and therefore the Higgs can be replaced by its VEV in the action. This also allows the results from this section to be directly carried over to Higgsless models [8]. 


\subsection{Massive Gauge Bosons}

We begin by considering the radion couplings to the massive gauge bosons of the SM, the $W^{ \pm}$and the $Z$. In our discussion, we shall focus exclusively on the $W^{ \pm}$, the generalization to the $Z$ being straightforward. In the limit when the effects of brane stabilization are neglected, the relevant terms in the action for the $W$ bosons take the form

$$
\mathcal{S}=\int d^{4} x d \theta \delta(\theta-\pi) \sqrt{-G_{v}}\left[-\frac{1}{4 g^{2}} G_{v}^{\mu \rho} G_{v}^{\nu \sigma} W_{\mu \nu} W_{\rho \sigma}+G_{v}^{\mu \nu} D_{\mu} H^{\dagger} D_{\nu} H\right],
$$

where $G_{v}$ is the induced metric on the visible brane and $g$ is the gauge coupling. Here we use the convention where the gauge covariant derivative is given by $D_{\mu}=\partial_{\mu}+A_{\mu}$, and the gauge coupling appears in the kinetic term of the gauge fields. When $H$ is replaced by its VEV in Eq. (3.8), the $W$ bosons acquire a mass. Expanding out the components of the metric in terms of the radion and graviton, we find that the radion couples to the $W^{ \pm}$as

$$
\frac{2 m_{W}^{2}}{g^{2}} \frac{\widetilde{\varphi}}{f} W_{\mu}^{+} W^{\mu-}
$$

where the index on $W$ is now raised by the $4 D$ Minkowski metric of flat space. Just as for the SM Higgs, at tree level the coupling of the radion is proportional to the mass of the field it is coupling to.

In the presence of the GW field, the gauge kinetic term on the brane is modified to

$$
\mathcal{L} \supseteq \delta(\theta-\pi) \sqrt{-G_{v}}\left[-\frac{1}{4 \hat{g}^{2}} G_{v}^{\mu \rho} G_{v}^{\nu \sigma} W_{\mu \nu} W_{\rho \sigma}\left(1+\alpha_{W} \frac{\Phi}{k^{3 / 2}}\right)\right] .
$$

Here $\hat{g}$ represents the gauge coupling in the absence of the GW stabilization mechanism, and $\alpha_{W}$ is a dimensionless number. We take $\alpha_{W}$ to be its natural value but continue to work in a regime where $v$ is small and work to leading order in $v$. This turns out to be equivalent to working to linear order in $\alpha_{W}$. The physical gauge coupling is now given by

$$
\frac{1}{4 g^{2}}=\frac{1}{4 \hat{g}^{2}}\left(1+\alpha_{W} \frac{\Phi(\pi)}{k^{3 / 2}}\right) \text {. }
$$

To incorporate radion fluctuations about the VEV of $\Phi$, we let

$$
\Phi(\pi) \rightarrow \Phi(\pi)\left(1+\frac{\partial_{\varphi} \Phi(\pi)}{\Phi(\pi)} \widetilde{\varphi}\right) .
$$

Using the classical solution for $\Phi$, the interaction term leads to a correction to the radion coupling. We consider first the case when the bulk potential for $\Phi$ is dominated by the mass term and the self-interactions can be neglected. In this limit, the radion coupling is given by

$$
\bar{c}_{W}\left(\epsilon v e^{-\epsilon k r_{c} \pi}\right) \frac{1}{4 g^{2}} \frac{\widetilde{\varphi}}{f} W_{\mu \nu} W^{\mu \nu} .
$$

Here $\bar{c}_{W}$ is an $\mathcal{O}(1)$ number, and indices on $W$ are raised by the Minkowski metric $\eta^{\mu \nu}$. Using the minimization condition and the formula for the mass of the radion, we have 
$v \epsilon e^{-\epsilon k r_{c} \pi} \sim \epsilon \tau \sim m_{\varphi}^{2} / m_{K K}^{2}$. Therefore the correction scales as $m_{\varphi}^{2} / m_{K K}^{2}$, and is small for a light radion. ${ }^{2}$

The GW scalar also couples to the gauge covariant kinetic term for the Higgs. These interactions affect the gauge boson masses, and lead to corrections to the radion couplings to these fields. We therefore consider the term

$$
\mathcal{L} \supseteq \delta(\theta-\pi) \sqrt{-G_{v}}\left[\left(1+\beta_{W} \frac{\Phi}{k^{3 / 2}}\right) G_{v}^{\mu \nu} D_{\mu} H^{\dagger} D_{\nu} H\right],
$$

where $\beta_{W}$ is a dimensionless number. Working in unitary gauge, we replace the Higgs field $H$ by its VEV. The physical gauge boson mass is now modified to

$$
m_{W}^{2}=\hat{m}_{W}^{2} \frac{\left(1+\beta_{W} \Phi(\pi) / k^{3 / 2}\right)}{\left(1+\alpha_{W} \Phi(\pi) / k^{3 / 2}\right)},
$$

where $\hat{m}_{W}$ is defined as the gauge boson mass in the absence of the GW stabilization mechanism. Including the radion fluctuations about the VEV of $\Phi$, we obtain for the radion coupling

$$
\frac{\widetilde{\varphi}}{f}\left[2+c_{W}\left(\epsilon v e^{-\epsilon k r_{c} \pi}\right)\right] \frac{m_{W}^{2}}{g^{2}} W_{\mu}^{+} W^{\mu-} .
$$

Here $c_{W}$ is an $\mathcal{O}(1)$ number. We see that this correction is also suppressed by $m_{\varphi}^{2} / m_{K K}^{2}$, and is small for a light radion.

For the case when the bulk potential for $\Phi$ has a cubic interaction and no mass term, we can repeat the steps above to obtain the corrections to the radion coupling. The end result however remains the same, with the corrections again scaling as $m_{\varphi}^{2} / m_{K K}^{2}$.

\subsection{Massless Gauge Bosons}

Next we consider the case of radion couplings to the massless gauge bosons of the SM, the photon and the gluons, on the IR brane. The kinetic term has the same form as Eq. (3.8), but now the coupling to the Higgs is absent. Expanding out the components of the metric in terms of the $4 D$ graviton and radion, we find that the radion does not couple to the massless gauge bosons. However this statement is true only at the classical level. In general, quantum effects generate a coupling of the massless gauge bosons to the radion at one loop. This can be understood as arising from quantum corrections to the trace of the energy momentum tensor; the trace anomaly. These contributions have been calculated in [23], and the resulting coupling is given by

$$
\frac{b_{<}}{32 \pi^{2}} \frac{\widetilde{\varphi}}{f} F_{\mu \nu} F^{\mu \nu}
$$

where again the indices are raised by $\eta^{\mu \nu}$. Here $b_{<}$is proportional to the one-loop $\beta$-function coefficient for the running of the gauge coupling,

$$
\frac{d}{d \log \mu} \frac{1}{g^{2}}=\frac{b_{<}}{8 \pi^{2}} .
$$

\footnotetext{
${ }^{2}$ We note that the operators in Eq. (3.9) and Eq. (3.13) are different, and that it may be possible to tell their contributions apart using gauge boson polarizations, especially at high energy.
} 
This formula is valid at the KK scale and must be renormalization group (RG) evolved to the radion mass to determine the couplings to the photon and gluons for the on-shell radion.

In the presence of the GW stabilization mechanism, there is a coupling between $\Phi$ and the gauge bosons given in Eq. (3.10), which modifies the gauge coupling as in Eq. (3.11). In the case where the GW scalar has a bulk mass term, a coupling between the radion and gauge bosons of the form of Eq. (3.13) is generated by the stabilization dynamics. This coupling is of order $m_{\varphi}^{2} / m_{K K}^{2}$, and, in the case where the bulk potential of $\Phi$ has no mass but a cubic interaction, it is straightforward to verify that the correction is of the same form and again scales as $m_{\varphi}^{2} / m_{K K}^{2}$. Although the corrections arising from stabilization are small, the fact that the leading order effect is loop suppressed implies that when the radion is only moderately lighter than the Kaluza-Klein scale, the effects of GW stabilization are significant, and may even dominate.

\subsection{Fermions}

We finally consider the case of the radion couplings to brane localized SM fermions. For concreteness we will focus on the radion couplings to up-type quarks, $Q$ and $U$. The generalization to the other fermions is straightforward. In the absence of a stabilization mechanism, the relevant part of the action has the form

$$
\mathcal{S}=\int d^{4} x d \theta \delta(\theta-\pi) \sqrt{-G_{v}}\left[\frac{i}{2} e_{a}^{\mu}\left(\bar{Q} \Gamma^{a} \overleftrightarrow{\partial_{\mu}} Q+\bar{U} \Gamma^{a} \overleftrightarrow{\partial_{\mu}} U\right)-y\left(\bar{Q} H U+\bar{U} H^{\dagger} Q\right)\right]
$$

where $\overleftrightarrow{\partial}=\vec{\partial}-\overleftarrow{\partial}$ and $e_{a}^{\mu}$ is the vierbein. We replace $H$ by its VEV and expand the components of the metric and vierbein out in terms of the $4 D$ graviton and radion. After expanding $\varphi$ about its VEV, canonically normalizing the kinetic terms of the fields $Q$ and $U$, and using the equations of motion for the fermions [58, 59], we obtain the coupling of the fermions to the radion as

$$
-m_{f} \frac{\widetilde{\varphi}}{f}(\bar{Q} U+h . c .)
$$

showing that the radion couples proportional to mass as expected.

The GW stabilization mechanism allows the following additional interaction terms involving $\Phi$,

$$
\mathcal{L}_{\text {int }}=\delta(\theta-\pi) \sqrt{-G_{v}} \frac{\Phi}{k^{3 / 2}}\left[\frac{i}{2} e_{a}^{\mu}\left(\alpha_{q} \bar{Q} \Gamma^{a} \overleftrightarrow{\partial_{\mu}} Q+\alpha_{u} \bar{U} \Gamma^{a} \overleftrightarrow{\partial_{\mu}} U\right)-\beta y\left(\bar{Q} H U+\bar{U} H^{\dagger} Q\right)\right]
$$

where $\alpha_{q}, \alpha_{u}$ and $\beta$ are dimensionless numbers. After $\Phi$ gets a VEV, the kinetic and mass terms receive corrections. The fluctuations of $\Phi$ about its VEV give rise to corrections to the radion couplings. We first focus on the case when the bulk potential for $\Phi$ consists only of a mass term. After making the kinetic terms canonical, and using the equations of 
motion for the fermions, the coupling to $\widetilde{\varphi}$ is determined to be of the form

$$
-m_{f} \frac{\widetilde{\varphi}}{f}(\bar{Q} U+h . c .)\left[1+c_{\psi} \epsilon v e^{-\epsilon k r_{c} \pi}\right] .
$$

Here $c_{\psi}$ is an $\mathcal{O}(1)$ number. We see that the corrections are suppressed by $\sim m_{\varphi}^{2} / m_{K K}^{2}$, and are therefore small for a light radion. In the case of a cubic bulk potential for $\Phi$, the corrections are of the same form and also suppressed by $\sim m_{\varphi}^{2} / m_{K K}^{2}$.

\section{The Dual Picture}

In this section, we apply the rules of the AdS/CFT dictionary to compare the results obtained in this paper for the mass and the couplings of the radion to those in the literature for its holographic dual, the dilaton [43, 44], and we find good agreement. In the process, we are also able to arrive at a holographic interpretation of our results for the radion. In particular, we will establish that the bulk self-interactions for $\Phi$ that seemed somewhat ad-hoc and non-minimal from the higher dimensional viewpoint, are well motivated in the dual picture, and are in fact necessary to obtain agreement. Our approach in this section will be to review the calculations for the mass and the couplings of the dilaton in a strongly coupled $\mathrm{CFT}$ and to compare against the corresponding results for the radion from earlier sections of this work.

In the AdS/CFT dictionary, the coordinate corresponding to the fifth dimension of AdS space is associated with the renormalization scale $\mu$ in the dual theory. To make this more precise, consider making a change of coordinates in AdS space from $\theta$ to $z$, where $z$ is defined as

$$
z=\frac{e^{k r_{c} \theta}}{k} .
$$

Then the renormalization scale $\mu$ in the dual CFT is given by $\mu \sim 1 / z$. Therefore, the RS set-up with two branes is dual to a strongly coupled theory that is well approximated by a CFT in the energy regime between the two branes. The hidden brane corresponds to the UV cut-off of the theory. The boundary conditions on the bulk fields at this boundary determine the coefficients of the deformation of the CFT in the UV, in the dual picture. The visible brane ends the AdS space in the IR, signaling the breakdown of the CFT. Various checks can be performed that are suggestive of a spontaneous breakdown. In particular, the trace of the energy momentum tensor is unchanged by the presence of the visible brane. Furthermore, the two point function of the dilatation current has a massless pole of the appropriate strength as dictated by Goldstone's theorem [32]. The scalar excitation associated with this massless pole, the radion, is identified with the NGB of broken scale invariance, the dilaton.

The AdS geometry is stabilized by adding a GW scalar $\Phi$ to the theory. In the dual picture, this corresponds to deforming the CFT by a primary scalar operator $\mathcal{O}$. The boundary condition for $\Phi$ on the UV brane is related to the strength of the deformation. In what follows, we compare the potentials for the radion and the dilaton on the two sides of the duality. We first focus on the original model where there is no stabilization mechanism 
before considering the scenario when the GW scalar is present. In both cases, we obtain good agreement. We make use of the ideas of holographic renormalization [60-66] when identifying parameters on the two sides of the correspondence.

\subsection{Dynamics in the Absence of a Stabilization Mechanism}

The dilaton is the NGB associated with the spontaneous breaking of conformal symmetry. Under the scale transformation $x^{\mu} \rightarrow x^{\prime \mu}=e^{-\omega} x^{\mu}$, the dilaton undergoes a shift, $\sigma(x) \rightarrow$ $\sigma^{\prime}\left(x^{\prime}\right)=\sigma(x)+\omega f$. Here $f$ is the scale associated with the breaking of conformal symmetry. For the purpose of writing interactions of the dilaton it is convenient to define the object

$$
\chi(x)=f e^{\sigma(x) / f},
$$

which transforms linearly under scale transformations. Specifically, under the scale transformation $x^{\mu} \rightarrow x^{\prime \mu}=e^{-\omega} x^{\mu}, \chi(x)$ transforms as a conformal compensator

$$
\chi(x) \rightarrow \chi^{\prime}\left(x^{\prime}\right)=e^{\omega} \chi(x) .
$$

This symmetry forbids a mass term for $\chi$. However, unlike the NGBs associated with a spontaneously broken internal symmetry, the potential for $\chi$ admits a quartic term

$$
V(\chi)=\kappa_{0} \frac{\chi^{4}}{4 !}
$$

Unless $\kappa_{0}$ is fine-tuned to zero, this potential does not admit a stable minimum away from the origin.

We now consider the radion potential, which is very similar. Before stabilization, $\varphi$ is massless in AdS and corresponds to the freedom to alter the distance between the two branes. The potential for $\varphi$ in the absence of stabilization is given by (see appendix A)

$$
V(\varphi)=\frac{k^{4}}{F^{4}} \tau \varphi^{4}
$$

We see that the forms of the potential for the dilaton and the radion are identical. On the AdS side, $\tau=\left(T_{v}-\Lambda_{b} / k\right) / k^{4}$, and we have

$$
\tau \Leftrightarrow \kappa_{0}
$$

where we use the double arrow to denote that these two quantities are related by the duality. In the original RS solution, the IR brane tension $T_{v}$ is chosen to set $\tau=0$. In the dual theory, this is equivalent to fine-tuning $\kappa_{0}$ to vanish.

\subsection{Dynamics in the Presence of a Stabilization Mechanism}

In the dual description, the addition of the GW scalar corresponds to deforming the CFT by a primary scalar operator $\mathcal{O}$ that has scaling dimension $\Delta$ in the far UV,

$$
\mathcal{L}_{C F T} \rightarrow \mathcal{L}_{C F T}+\hat{\lambda}(\mu) \mu^{4-\Delta} \mathcal{O}(x) .
$$


Here $\hat{\lambda}(\mu)$ is the renormalized coupling constant. Defined in this way it is dimensionless. We choose to normalize the operator $\mathcal{O}$ such that $\hat{\lambda}$ of order one corresponds to conformal symmetry violation becoming strong, so that it can no longer be treated as a perturbation. If the operator $\mathcal{O}$ is relevant $(\Delta<4)$, the deformation at the UV scale $\Lambda_{\mathrm{UV}}$ grows in the IR leading to the breaking of the CFT at the IR scale $f$. Below the breaking scale the spectrum contains the dilaton $\chi$, which is the Goldstone boson of spontaneously broken conformal symmetry. As a consequence of the explicit conformal symmetry violation associated with the operator $\mathcal{O}$, the potential for $\chi$ gets modified from Eq. (4.4). This results in $\chi$ acquiring a mass. The potential for the dilaton $\chi$ in the presence of this explicit breaking of CFT can be determined from a spurion analysis. In the presence of the deformation, the coefficient $\hat{\lambda}$ satisfies a renormalization group (RG) equation above the symmetry breaking scale

$$
\frac{d \log \hat{\lambda}}{d \log \mu}=-g(\hat{\lambda})
$$

where $g(\hat{\lambda})$ is a polynomial in $\hat{\lambda}$ that can be parametrized as

$$
g(\hat{\lambda})=(4-\Delta)+c_{1} \hat{\lambda}+c_{2} \hat{\lambda}^{2}+\ldots
$$

With our normalization of the operator $\mathcal{O}$, the coefficients $c_{i}$ are expected to be of order one.

The cases of interest for electroweak symmetry breaking correspond to those where there is a large hierarchy between the cutoff scale $\Lambda_{\mathrm{UV}}$ and the scale $f$ where conformal symmetry is broken. A large hierarchy can be generated if the deformation $\hat{\lambda}$ is exponentially small in the UV. This is technically natural if the operator $\mathcal{O}$ breaks a symmetry of the CFT, and is therefore protected. The AdS/CFT correspondence relates this scenario to the case when the value of $\Phi$ at the UV brane is exponentially small. Alternatively, a large hierarchy can be generated if the operator $\mathcal{O}$ is close to marginal in the UV, so that $|4-\Delta| \ll 1$. Duality relates this scenario to the case when the bulk mass term for the GW scalar is small in units of the curvature. It is this latter scenario which we will focus on.

We wish to determine the form of the potential for the dilaton in the low energy effective theory. To do this, we note that the UV theory possesses a spurious scale invariance if $\hat{\lambda}$ is assigned a (spurious) scaling dimension $g(\hat{\lambda})$. The potential for the dilaton in the low energy theory must respect this spurious symmetry. This is a severe restriction on the possible terms that can appear.

For the purposes of obtaining the low energy effective theory it is useful to construct an object $\bar{\Omega}(\hat{\lambda}, \chi / \mu)$ that is invariant under both infinitesimal scale and RG transformations,

$$
\bar{\Omega}(\hat{\lambda}, \chi / \mu)=\hat{\lambda}(1-g(\hat{\lambda}) \log (\chi / \mu)) .
$$

For values of $\mu$ close to the symmetry breaking scale $f$ and for small $g(\hat{\lambda})$, this invariant combination can be approximated as

$$
\bar{\Omega}(\hat{\lambda}, \chi / \mu) \approx \hat{\lambda}\left(\frac{\chi}{f}\right)^{-g(\hat{\lambda})},
$$


where $\hat{\lambda}$ in this expression is understood to be evaluated at $\mu=f$. Then by requiring invariance under spurious scale transformations we can obtain the potential for the dilaton. To leading order in $\hat{\lambda}$ it is given by [43]

$$
V(\chi)=\frac{\chi^{4}}{4 !}\left[\kappa_{0}-\kappa_{1} \bar{\Omega}(\hat{\lambda}, \chi / \mu)\right] \approx \frac{\chi^{4}}{4 !}\left[\kappa_{0}-\kappa_{1} \hat{\lambda}\left(\frac{\chi}{f}\right)^{-g(\hat{\lambda})}\right] .
$$

Here $\kappa_{0}$ and $\kappa_{1}$ are parameters in the low energy theory. The potential now admits a stable minimum at $\langle\chi\rangle=f$ without fine-tuning $\kappa_{0}$ to zero. For $g(\hat{\lambda}) \ll 1$, the minimization condition takes the form,

$$
\kappa_{0}=\kappa_{1} \hat{\lambda}
$$

In this equation, $\hat{\lambda}$ is evaluated at the breaking scale $f$. This implicitly determines the breaking scale. By minimizing this potential, the mass of the dilaton $\sigma$ can be obtained as

$$
m_{\sigma}^{2}=\frac{4 \kappa_{0}}{4 !} g(\hat{\lambda}) f^{2}
$$

It follows that the mass of the dilaton depends on $\kappa_{0}$ and $g(\hat{\lambda})$, which are related to each other by Eqs. (4.9) and (4.13). We focus on the case when $\kappa_{0}$ lies below its natural strong coupling value by a factor which could be as small as order a few. However the other parameters including $\kappa_{1}$ and the $c_{i}$ are assumed to be of order their natural values. In this limit, $\hat{\lambda}$ at the minimum is also expected to lie below its strong coupling value, and by roughly the same factor. For sufficiently small $\kappa_{0}, g(\hat{\lambda})$ at the minimum is dominated by the constant term in Eq. (4.9) so so that it scales as $g(\hat{\lambda}) \sim(4-\Delta) \ll 1$. In this limit the mass squared of the dilaton scales as $m_{\sigma}^{2} \sim \kappa_{0}(4-\Delta)$.

However, since in the theories of interest we have $4-\Delta \ll 1$, for larger values of $\kappa_{0}$ we expect that close to the breaking scale $\mu=f$ the term linear in $\hat{\lambda}$ in Eq. (4.9) will dominate over the constant term. In this limit $g(\hat{\lambda})$ at the minimum scales as $g(\hat{\lambda}) \sim \hat{\lambda} \sim \kappa_{0}$. We then have $g(\hat{\lambda}) \sim \kappa_{0}$, so that in this limit the mass squared scales as $m_{\sigma}^{2} \sim \kappa_{0}^{2}$. Hence we see that, depending on the extent to which $\kappa_{0}$ lies below its natural strong coupling value, the dilaton mass may scale either as $\sqrt{\kappa_{0}(4-\Delta)}$ or as $\kappa_{0}$. Recall that duality relates $\kappa_{0}$ to $\tau$, the detuning of the IR brane tension. We therefore see that the results for the scaling of the radion mass with the IR brane tension are indeed reproduced on the CFT side of the correspondence.

Following this quick review we are ready to draw more detailed parallels to the AdS side of the duality in the presence of stabilization. Recall that the classical equation satisfied by $\Phi$ has two independent solutions, which in the limit that $k r_{c} \gg 1$, are well approximated by the OR and BR solutions defined in section 2. The OR differential equation is simply first order, and is a good approximation to the classical equation that is satisfied by $\Phi$ everywhere except in the boundary layer region close to the IR brane. In the absence of an IR brane, the AdS/CFT dictionary relates the value of $\Phi$ at the UV brane to the coefficient of the deformation of the CFT, $\hat{\lambda}$, evaluated at the renormalization scale $\mu=k[29,30,32]$. We denote this correspondence by

$$
\frac{\Phi(\theta=0)}{k^{3 / 2}} \Leftrightarrow \hat{\lambda}\left(\Lambda_{\mathrm{UV}}\right)
$$


The ideas of holographic renormalization extend this identification further. In the AdS theory without an IR brane, the BR solution is replaced by the requirement of regularity at the AdS horizon. In that case, the value of $\Phi$ at an arbitrary point with coordinates $\left(x^{\mu}, \theta\right)$ in the bulk corresponds to $\hat{\lambda}$ evaluated at the renormalization scale $\mu=k \exp \left(-k r_{c} \theta\right)$. This then implies that the first order OR differential equation for $\Phi$ corresponds to the renormalization group equation (RGE) for $\hat{\lambda}$ at energies below the UV cutoff.

In our case, the AdS space has an IR brane and we cannot discard the BR solution, which captures the physics associated with the conformal symmetry breaking phase transition. However, given that the boundary layer has a thickness of just $\sim 1 / 4 k r_{c} \sim \epsilon$ in $\theta$ coordinates, as long as we are at least this distance from the IR brane, the correspondence between the OR equation for $\Phi$ and the RG equation for $\hat{\lambda}$ holds. From Eq. (2.14), the OR equation satisfied by $\Phi$ is

$$
\frac{d \log \Phi_{O R}}{d\left(k r_{c} \theta\right)}=-\frac{m^{2}}{4 k^{2}}-\frac{\eta}{8 \sqrt{k}} \frac{\Phi_{O R}}{k^{3 / 2}}-\frac{\zeta k}{24} \frac{\Phi_{O R}^{2}}{k^{3}}+\ldots .
$$

This result agrees with the equation obtained using conventional Hamilton-Jacobi methods, in the limit that $m^{2} / 4 k^{2} \ll 1[63]$.

The corresponding RG equation satisfied by the coefficient of the deformation $\hat{\lambda}$ is given in Eqs. (4.8) and (4.9). From the AdS/CFT dictionary, we have that the coordinate in the fifth dimension $\theta$ corresponds to the renormalization scale $\mu$ in the $4 D$ theory, $k \exp \left(-k r_{c} \theta\right)=\mu$. While it is tempting to use this to relate the various terms in Eq. (4.16) and Eq. (4.9), we are prevented from doing so by the fact that RGEs are in general scheme dependent. However, because this restriction does not apply to the lowest order term in an RGE, we are able to reproduce the familiar AdS/CFT relation between the dimensions of operators in the $4 D$ theory and the masses of the corresponding scalar fields in $5 \mathrm{D}$, $\Delta=4+m^{2} / 4 k^{2}=4+\epsilon$ for $\epsilon \ll 1$. In addition, in the case when the bulk mass term is small so that the potential for the GW scalar is dominated by the cubic self-interaction term, we can also equate

$$
c_{1} \hat{\lambda}=-\frac{\eta}{8 \sqrt{k}} \frac{\Phi_{O R}}{k^{3 / 2}}
$$

Comparing the RGE for $\hat{\lambda}$ and the OR differential equation for $\Phi$, it is clear that the limit when $\hat{\lambda}$ is small and $g(\hat{\lambda})$ is dominated by the constant term corresponds to the AdS potential for $\Phi$ being dominated by the mass term. For larger values of $\hat{\lambda}$, the linear and higher order terms in $g(\hat{\lambda})$ dominate. This corresponds to the cubic and higher order self-interaction terms becoming important in the bulk potential for $\Phi$.

The potential for the dilaton is to be compared to that for the radion in the two cases that we studied in section 2. Consider first the case when the self-interactions for $\Phi$ in the bulk can be neglected. In this limit, $g(\hat{\lambda})$ in the RG equation for $\hat{\lambda}$ is dominated by the constant term. The potential for the dilaton using Eq. (4.12) is then given by

$$
V(\chi)=\frac{\chi^{4}}{4 !}\left[\kappa_{0}-\kappa_{1} \hat{\lambda}\left(\frac{\chi}{f}\right)^{\Delta-4}\right] .
$$


The resulting potential for the radion from Eq. (2.21) is given by

$$
V(\varphi)=\frac{k^{4}}{F^{4}} \varphi^{4}\left[\tau+\frac{2 \alpha v}{F^{\epsilon}} \varphi^{\epsilon}\right]=\frac{k^{4}}{F^{4}} \varphi^{4}\left[\tau+\frac{2 \alpha\left(v e^{-\epsilon k r_{c} \pi}\right)}{\left(F e^{-k r_{c} \pi}\right)^{\epsilon}} \varphi^{\epsilon}\right],
$$

where we have rewritten it in a form that is convenient for making the comparison. Bearing in mind that the symmetry breaking scale $f$ is of order $k e^{-k r_{c} \pi}$, the duality allows us to relate

$$
\begin{aligned}
\hat{\lambda}(f) & \Leftrightarrow \frac{\Phi_{O R}(\theta \sim \pi)}{k^{3 / 2}} \approx v e^{-\epsilon k r_{c} \pi} \\
\Delta & =4+\epsilon .
\end{aligned}
$$

With this identification, the potentials for the radion and the dilaton are of the same form. The low energy parameter $\kappa_{1}$ on the CFT side is related to the coefficient $\alpha$ of the potential for $\Phi$ on the visible brane. Our initial identification $\kappa_{0} \Leftrightarrow \tau$ continues to hold even though $\tau$ now receives additional $\mathcal{O}(1)$ contributions from visible brane dynamics. Since the potentials for $\varphi$ and $\chi$ are of the same form, the leading order expression for the masses of the fluctuations of $\varphi$ and $\chi$ have the same parametric dependence.

Consider next the scenario when the potential for the GW scalar $\Phi$ is dominated by the cubic self-interaction term in the bulk. By duality this is related to the case when $g(\hat{\lambda})$ in the RG equation for $\hat{\lambda}$ is dominated by the linear term $c_{1} \hat{\lambda}$. We can read off the potential for the dilaton from Eq. (4.12). In this limit,

$$
V(\chi)=\frac{\chi^{4}}{4 !}\left[\kappa_{0}-\kappa_{1} \hat{\lambda}\left(\frac{\chi}{f}\right)^{-c_{1} \hat{\lambda}}\right] .
$$

In the corresponding limit, we obtain the potential for the radion from Eq. (2.30) as

$$
V(\varphi)=\frac{k^{4}}{F^{4}} \varphi^{4}\left[\tau+\frac{2 \alpha v}{1-\xi \log (\varphi / F)}\right]=\frac{k^{4}}{F^{4}} \varphi^{4}\left[\tau+\frac{2 \alpha v}{1+\xi k r_{c} \pi-\xi \log \left(\varphi / F e^{-k r_{c} \pi}\right)}\right],
$$

where $\xi=\eta v / 8 \sqrt{k}$. In the second equality we have added and subtracted $\xi k r_{c} \pi$ in the denominator. For $\xi /\left(1+\xi k r_{c} \pi\right) \ll 1$, we can approximate the potential as

$$
V(\varphi) \approx \varphi^{4}\left[\tau+\frac{2 \alpha v}{1+\xi k r_{c} \pi}\left(\frac{\varphi}{F e^{-k r_{c} \pi}}\right)^{\frac{\xi}{1+\xi k r_{c} \pi}}\right] .
$$

This is a more convenient form to compare against the dilaton potential. In the limit in which we are working, $f$ is of order $k e^{-k r_{c} \pi}$. The duality allows us to relate

$$
\begin{aligned}
\hat{\lambda}(f) & \Leftrightarrow \frac{v}{1+\xi k r_{c} \pi} \\
c_{1} \hat{\lambda}(f) & =-\frac{\xi}{1+\xi k r_{c} \pi} .
\end{aligned}
$$

We once again see that the potentials for the radion and the dilaton are of the same form. The low energy parameters in the CFT, $\kappa_{0}$ and $\kappa_{1}$ are again related to the AdS parameters 
$\tau$ and $\alpha$ respectively. The parametric dependences of the masses of the radion and the dilaton then agree in a straightforward manner.

Finally, we can use the correspondence from Eq. (4.15) to get a better understanding of the behavior of $\Phi(\theta)$ shown in Fig. 1. Since $\theta$ is dual to $\log \mu$, we see that in the outer region, which corresponds to most of the space, the coupling runs logarithmically as we would expect for a nearly marginal deformation of the CFT. Near the IR brane at $\theta=\pi$, the behavior of $\Phi$ changes due to the formation of a boundary layer. This is the region where the behavior of $\Phi(\theta)$ can no longer be described by the OR Eq. (2.14) and we need a different description. In the dual picture, this corresponds to the phase transition associated with the spontaneous breakdown of the CFT in the IR, caused by the deformation growing large. Thus we see that in the outer region, Fig. 1 is also a good description of the behavior of $\hat{\lambda}(\mu)$, the coefficient of the deformation in the dual theory.

\section{Conclusion}

In this paper we have constructed the effective field theory for a radion which is stabilized by the GW mechanism, but which remains light compared to the KK scale of the extra dimension. Our analysis differs from the original GW construction in that we do not tune the gravitational potential for the radion to vanish, but allow an interplay between the dynamics of the GW scalar and gravity to stabilize the radion. We require that the bulk mass for the GW scalar $\Phi$ is small compared to the inverse curvature scale in order to generate a large hierarchy between the scales of the hidden and visible branes.

We consider two different cases for the bulk potential of the GW scalar. In the first, the mass for $\Phi$, although small, is still the dominant term in the potential. This is the case most often studied in the literature, and we find that the radion is light. In particular, the smallness of the bulk mass in units of the inverse AdS curvature translates to radion being lighter than the KK scale. However, the assumption that the bulk potential is dominated by the mass term is not expected to be satisfied in the general case of theories with a CFT dual. Relaxing this assumption, we consider the second case when the bulk potential for $\Phi$ is dominated by a cubic term. This captures the features of a general interacting potential. We take the coefficient of the cubic coupling to be around its strong coupling value. This case differs from the previous one because the radion is generically not light. On the other hand, its mass is controlled by the visible brane tension which can be tuned to allow the radion to lie below the KK scale, even if all the GW interactions are at their strong coupling values. The tuning is mild, scaling as the mass of the radion rather than as the square of the mass.

We have analyzed our results in light of the AdS/CFT correspondence, exploring the connection to the recent results in the literature for a light dilaton. We focused on CFTs which are deformed by marginal operators that are small in the UV, but grow large in the IR to trigger the breaking of conformal symmetry. In general, the scaling behavior of these operators near the breaking scale is very different from their scaling behavior in the UV. The AdS/CFT dictionary associates this change in scaling behavior with the presence of self-interaction terms for the Goldberger-Wise scalar $\Phi$ in the bulk. It follows from this 
that the inclusion of bulk self-interactions for $\Phi$, such as the cubic coupling considered here, are necessary to describe the properties of the radion in RS theories with holographic duals. Our results for the radion are in good agreement with those obtained earlier for the dilaton. In particular, we find that the presence of a light radion is not a robust prediction of RS models with a holographic dual, but is instead associated with mild tuning.

Finally, we have analyzed radion couplings to SM fields living on the visible brane, focusing in particular on corrections due to the GW mechanism. These corrections are proportional to the mass squared of the radion in units of the KK scale, and are small if the radion is light. Because in the classical limit the radion coupling to each SM field is proportional to some power of its mass, the corrections from stabilization are subleading for massive SM fields, but can be important and possibly dominant for the photon and the gluon.

\section{Acknowledgments}

We would like to thank Raman Sundrum for many invaluable discussions. ZC and RKM are supported by the NSF under grant PHY-0968854. DS is supported in part by the NSF under grant PHY-0910467 and gratefully acknowledges support from the Maryland Center for Fundamental Physics.

\section{A $5 D$ Gravity without a GW Scalar}

We give here the intermediate steps in obtaining the effective $4 D$ action that includes the $4 D$ graviton and the radion fields. In the absence of a stabilization mechanism, the $5 D$ action is

$$
\mathcal{S}_{G R}^{5 D}=\int d^{4} x \int_{-\pi}^{\pi} d \theta \sqrt{G}\left(-2 M_{5}^{3} \mathcal{R}[G]-\Lambda_{b}\right)-\sqrt{-G_{h}} \delta(\theta) T_{h}-\sqrt{-G_{v}} \delta(\theta-\pi) T_{v},
$$

where the geometry is a slice of $\mathrm{AdS}_{5}$ compactified on a circle $\mathcal{S}_{1}$ with $\mathcal{Z}_{2}$ orbifolding. The metric is then given as

$$
d s^{2}=e^{-2 k r(x)|\theta|} g_{\mu \nu} d x^{\mu} d x^{\nu}-r^{2}(x) d \theta^{2},
$$

where $g_{\mu \nu}$ is the $4 D$ graviton and $r(x)$ is the (non-canonical) radion. The $5 D$ Ricci scalar for this metric is

$$
\begin{aligned}
\mathcal{R}[G]=\frac{2}{r(x)}\left[e^{2 k r(x)|\theta|}\right. & \left(-\frac{r}{2} \mathcal{R}[g]-\partial^{2} r+3 k r|\theta| \partial^{2} r+2 k|\theta| \partial r \partial r-3 k^{2} r|\theta|^{2} \partial r \partial r\right) \\
& \left.+10 k^{2} r-8 k \delta(\theta)+8 k \delta(\theta-\pi)\right] .
\end{aligned}
$$

Using $\sqrt{G}=r(x) e^{-4 k r(x)|\theta|} \sqrt{-g}$, the integrand in the action is

$$
\begin{aligned}
& \sqrt{-g} e^{-2 k r(x)|\theta|}\left[2 M_{5}^{3}\left(r \mathcal{R}[g]+2 \partial^{2} r-4 k|\theta| \partial r \partial r-6 k r|\theta| \partial^{2} r+6 k^{2} r|\theta|^{2} \partial r \partial r\right)\right] \\
& +\sqrt{-g} e^{-4 k r(x)|\theta|}\left[-r \Lambda_{b}+2 M_{5}^{3}\left(-20 k^{2} r+16 k \delta(\theta)-16 k \delta(\theta-\pi)\right)-T_{h} \delta(\theta)-T_{v} \delta(\theta-\pi)\right] .
\end{aligned}
$$


Using integration by parts, the integrand further reduces to

$$
\begin{aligned}
& \sqrt{-g} e^{-2 k r(x)|\theta|}\left[2 M_{5}^{3}\left(r \mathcal{R}[g]+6 k|\theta| \partial r \partial r-6 k^{2}|\theta|^{2} r \partial r \partial r\right)\right] \\
& +\sqrt{-g} e^{-4 k r(x)|\theta|}\left[-r \Lambda_{b}+2 M_{5}^{3}\left(-20 k^{2} r+16 k \delta(\theta)-16 k \delta(\theta-\pi)\right)-T_{h} \delta(\theta)-T_{v} \delta(\theta-\pi)\right] .
\end{aligned}
$$

Integrating over $\theta$ leads to a cancellation between the $|\theta|$ and the $|\theta|^{2}$ terms as

$$
\int_{-\pi}^{\pi} d \theta e^{-2 k r|\theta|}\left(6 k|\theta|-6 k^{2} r|\theta|^{2}\right)=6 k \pi^{2} e^{-2 k r \pi}
$$

and leads to the effective action for the $4 D$ graviton $g_{\mu \nu}(x)$ and the modulus field $r(x)$ as

$$
\begin{aligned}
\mathcal{S}_{G R}^{4 D}=\frac{2 M_{5}^{3}}{k} & \int d^{4} x \sqrt{-g}\left(1-e^{-2 k \pi r(x)}\right) \mathcal{R}[g]+\frac{12 M_{5}^{3}}{k} \int d^{4} x \sqrt{-g} \partial_{\mu}\left(e^{-k \pi r(x)}\right) \partial^{\mu}\left(e^{-k \pi r(x)}\right) \\
& -\int d^{4} x \sqrt{-g} V(r) .
\end{aligned}
$$

The potential $V(r)$ for the modulus field has contributions both from bulk and brane, and is given by

$$
\begin{aligned}
V(r) & =-\int_{-\pi}^{\pi} d \theta e^{-4 k r(x)|\theta|}\left(-r \Lambda_{b}-40 M_{5}^{3} k^{2} r\right)+\left(T_{h}-32 M_{5}^{3} k\right)+\left(T_{v}+32 M_{5}^{3} k\right) e^{-4 k r(x) \pi} \\
& =e^{-4 k r(x) \pi}\left(T_{v}+32 M_{5}^{3} k-2 \frac{r \Lambda_{b}+40 M_{5}^{3} k^{2} r}{4 k r}\right)+\left(T_{h}-32 M_{5}^{3} k+2 \frac{r \Lambda_{b}+40 M_{5}^{3} k^{2} r}{4 k r}\right) .
\end{aligned}
$$

In terms of the canonically normalized $4 D$ radion field $\varphi \equiv F e^{-k \pi r(x)}$, where $F=\sqrt{24 M_{5}^{3} / k}$, the $4 D$ action looks like

$$
\begin{aligned}
\mathcal{S}_{G R}^{4 D}= & 2 M_{5}^{3} / k \int d^{4} x \sqrt{-g}\left(1-(\varphi / F)^{2}\right) \mathcal{R}+\frac{1}{2} \int d^{4} x \sqrt{-g} \partial_{\mu} \varphi \partial^{\mu} \varphi \\
& -\int d^{4} x \sqrt{-g}\left[(\varphi / F)^{4}\left(T_{v}-\frac{\Lambda_{b}}{2 k}+12 M_{5}^{3} k\right)+\left(T_{h}+\frac{\Lambda_{b}}{2 k}-12 M_{5}^{3} k\right)\right] .
\end{aligned}
$$

We ignore the interactions between the Ricci scalar and radion because they are small and irrelevant for phenomenology. Using $k^{2}=-\Lambda_{b} / 24 M_{5}^{3}$, the radion potential is given as

$$
V(\varphi)=\left[(\varphi / F)^{4}\left(T_{v}-\frac{\Lambda_{b}}{k}\right)+\left(T_{h}+\frac{\Lambda_{b}}{k}\right)\right] \equiv \frac{k^{4}}{F^{4}}\left(\tau \varphi^{4}+\varrho\right),
$$

where $\varrho=\left(T_{h}+\frac{\Lambda_{b}}{k}\right) / k^{4}$ is the $4 D$ cosmological constant which we tune to be small, and $\tau=\left(T_{v}-\frac{\Lambda_{b}}{k}\right) / k^{4}$. When the GW scalar is included, $\tau$ will receive additional corrections.

\section{B Radion Mass}

We give here the intermediate steps involved in obtaining the potential for the radion, the minimization condition and the mass of the radion. We consider two cases for the bulk 
potential $V_{b}(\Phi)$ : (i) the bulk potential dominated by a mass term and (ii) the bulk potential dominated by a cubic term. We will give the steps for obtaining the radion potential in both the cases. In the case where the bulk potential only has a mass term, the equation for $\Phi$ admits exact solution. We compare the approximate OR and BR solutions for $\Phi$ obtained earlier to the exact solution of $\Phi$ in this case.

\section{GW Scalar without Bulk Interactions}

Consider first the case of

$$
V_{b}(\Phi)=\frac{1}{2} m^{2} \Phi^{2}
$$

The equation satisfied by $\Phi$ in the bulk becomes

$$
\partial_{\theta}^{2} \Phi-4 k r_{c} \partial_{\theta} \Phi-m^{2} r_{c}^{2} \Phi=0 .
$$

The equation being a homogeneous second order differential equation, admits exact solutions given by

$$
\Phi(\theta, r)=A e^{\nu_{1} k r_{c}|\theta|}+B e^{\nu_{2} k r_{c}|\theta|},
$$

where $\nu_{1,2}=2 \pm \sqrt{4+m^{2} / k^{2}}$ and $A, B$ are constants, determined by the brane potentials as

$$
\begin{aligned}
A \nu_{1}+B \nu_{2} & =\frac{1}{2 k} V_{h}^{\prime}(\Phi) \\
\nu_{1} A e^{\nu_{1} k r_{c} \pi}+\nu_{2} B e^{\nu_{2} k r_{c} \pi} & =-\frac{1}{2 k} V_{v}^{\prime}(\Phi)
\end{aligned}
$$

Without specifying the form of $V_{h}$, we require that the value of $\Phi(\theta=0)=k^{3 / 2} v$. For the visible brane, we assume a simple form $V_{v}(\Phi)=2 k^{5 / 2} \alpha \Phi$. This gives

$$
\begin{gathered}
A+B=k^{3 / 2} v \\
\nu_{1} A e^{\nu_{1} k r_{c} \pi}+\nu_{2} B e^{\nu_{2} k r_{c} \pi}=-k^{3 / 2} \alpha .
\end{gathered}
$$

We take $\epsilon=m^{2} / 4 k^{2} \ll 1$ and $e^{-k r_{c} \pi} \ll 1$ but $e^{ \pm \epsilon k r_{c} \pi}$ unhierarchical. The two conditions on $A, B$ fix them to be

$$
\begin{aligned}
& A=k^{3 / 2} \frac{v \nu_{2} e^{\nu_{2} k r_{c} \pi}+\alpha}{\nu_{2} e^{\nu_{2} k r_{c} \pi}-\nu_{1} e^{\nu_{1} k r_{c} \pi}} \approx-\frac{k^{3 / 2} \alpha}{4} e^{-(4+\epsilon) k r_{c} \pi} \\
& B=k^{3 / 2} \frac{v \nu_{1} e^{\nu_{1} k r_{c} \pi}+\alpha}{\nu_{1} e^{\nu_{1} k r_{c} \pi}-\nu_{2} e^{\nu_{2} k r_{c} \pi}} \approx k^{3 / 2} v .
\end{aligned}
$$

Therefore, the leading order solution of $\Phi$ is given as

$$
\Phi(\theta)=-\frac{k^{3 / 2} \alpha}{4} e^{(4+\epsilon) k r_{c}(\theta-\pi)}+k^{3 / 2} v e^{-\epsilon k r_{c} \theta} .
$$

Using the arguments from section 2, an approximate solution for $\Phi$ can be obtained by a boundary layer analysis:

$$
\Phi_{\text {approx }}(\theta)=-\frac{k^{3 / 2} \alpha}{4} e^{4 k r_{c}(\theta-\pi)}+k^{3 / 2} v e^{-\epsilon k r_{c} \theta} .
$$

Figure 1 in section 2 shows that the approximate solution agrees very well with the exact solution. 


\section{Radion Potential}

Substituting either solution of $\Phi$ in the action, integrating over the extra dimension and letting $r_{c} \rightarrow r(x)$ generates a potential for the radion. The potential gets contribution both from the bulk and the brane terms in the GW action, and is given in general by

$$
V_{G W}(r)=\int_{0}^{\pi} d \theta \frac{1}{r} e^{-4 k r \theta}\left(\partial_{\theta} \Phi \partial_{\theta} \Phi+r^{2} m^{2} \Phi^{2}\right)+e^{-4 k r \pi} 2 \alpha k^{5 / 2} \Phi(\pi) .
$$

Working in the limit where $|\epsilon| \ll 1$, the bulk contribution to the potential goes as

$$
\begin{aligned}
V_{G W}^{\mathrm{bulk}}(r) & =k\left[(4+\epsilon) A^{2}\left(e^{\left(2 \nu_{1}-4\right) k r \pi}-1\right)-\epsilon B^{2}\left(e^{\left(2 \nu_{2}-4\right) k r \pi}-1\right)\right] \\
& =k^{4} \frac{e^{-4 k r \pi}}{4+\epsilon}\left(\alpha-\epsilon v e^{-\epsilon k r_{c} \pi}\right)^{2}-\epsilon k^{4} v^{2} e^{-(4+2 \epsilon) k r_{c} \pi}+\epsilon k^{4} v^{2}
\end{aligned}
$$

The brane contribution to the potential is

$$
\begin{aligned}
V_{G W}^{\text {brane }}(r) & =2 k^{5 / 2} \alpha e^{-4 k r \pi} \Phi(\pi) \\
& =k^{4} e^{-4 k r \pi} 2 \alpha\left[-\frac{\alpha-\epsilon v e^{-\epsilon k r_{c} \pi}}{4+\epsilon}+v e^{-\epsilon k r_{c} \pi}\right] .
\end{aligned}
$$

Keeping to linear order in $v$ and $\epsilon$ we find that the bulk contribution to the potential is subleading. We can now write the leading radion potential, including the gravitational contributions from Eq. (A.10), in terms of the canonical field $\varphi$ :

$$
V(\varphi)=k^{4}\left(\frac{\varphi}{F}\right)^{4}\left[\tau+2 \alpha v\left(\frac{\varphi}{F}\right)^{\epsilon}\right] .
$$

Here, $\tau$ receives contributions from both the gravity sector and the visible brane dynamics, and, to leading order in $\epsilon$, it is given by

$$
\tau=\frac{T_{v}-\Lambda_{b} / k-k^{4} \alpha^{2} / 4}{k^{4}} .
$$

The minimization condition gives $\langle\varphi\rangle=f$ as

$$
\tau+2 \alpha v\left(\frac{f}{F}\right)^{\epsilon}=0
$$

At the minimum, the mass squared is given as

$$
m_{\varphi}^{2}=-\frac{\epsilon \tau}{6} \frac{k^{3}}{M_{5}^{3}}\left(k e^{-k r_{c} \pi}\right)^{2}
$$

Including higher order terms does not change the parametric dependences.

\section{GW Scalar with Bulk Cubic Self Interaction}

We next consider the case of

$$
V_{b}(\Phi)=\frac{1}{3 !} \eta \Phi^{3}
$$


In this case, the classical equation satisfied by $\Phi$ is given as

$$
\partial_{\theta}^{2} \Phi-4 k r_{c} \partial_{\theta} \Phi-\frac{\eta}{2} r_{c}^{2} \Phi^{2}=0
$$

In the absence of an exact solution, we use the boundary layer theory described in section 2.1 to get the approximate solution for $\Phi$ as

$$
\Phi_{\text {approx }}(\theta)=-\frac{k^{3 / 2} \alpha}{4} e^{4 k r_{c}(\theta-\pi)}+\frac{k^{3 / 2} v}{1+\xi k r \theta}
$$

where $\xi=\eta v / 8 \sqrt{k}$.

The radion potential coming from the GW action with a cubic interaction in the bulk is given by

$$
V_{G W}(r)=\int_{0}^{\pi} d \theta \frac{1}{r} e^{-4 k r \theta}\left(\partial_{\theta} \Phi \partial_{\theta} \Phi+\frac{r^{2} \eta}{3} \Phi^{3}\right)+e^{-4 k r \pi} 2 \alpha k^{5 / 2} \Phi(\pi) .
$$

We now plug the solution from Eq. (B.18) into Eq. (B.19) to get an explicit form of the radion potential. Including the gravitational contribution and working to leading order in $v$ we get

$$
V(\varphi)=k^{4}\left(\frac{\varphi}{F}\right)^{4}\left[\tau+\frac{1}{1-\xi \log (\varphi / F)}\left(2 \alpha v+\frac{\alpha^{2} \xi}{8}\right)\right] .
$$

We define $w=2 \alpha v+\alpha^{2} \xi / 8$. The first term in $w$ comes from the brane potential while the second term comes from the bulk, and we note that both are small because they are proportional to $v$.

The potential is minimized at $\langle\varphi\rangle=f$ as

$$
\tau+\frac{w}{1-\xi \log (f / F)}=0
$$

The mass squared at the minimum is given by

$$
m_{\varphi}^{2}=\frac{\tau^{2} \xi}{6 w} \frac{k^{3}}{M_{5}^{3}}\left(k e^{-k r_{c} \pi}\right)^{2}
$$

At the NDA values of the parameters (see the next section), we find that $m_{\varphi}^{2} / m_{K K}^{2} \sim \tau^{2}$.

\section{NDA Estimation of Parameters}

In this appendix we give a brief review of estimates using naive dimensional analysis (NDA) [67, 68] and its application in five dimensions [69] as in this work. The general idea of NDA is to estimate the size of Lagrangian parameters by assuming that quantum corrections are the same size at every loop order.

The loop factor $\ell_{D}$ that comes from integrating over $D$-dimensional phase space is given by

$$
\ell_{D}=2^{D} \pi^{D / 2} \Gamma(D / 2),
$$


giving the familiar $\ell_{4}=16 \pi^{2}$. The relevant number for five dimensions is $\ell_{5}=24 \pi^{3}$. If there are $N$ states in the theory which go around in loops, then each loop contribution gets multiplied by $N$. For our case $N$ is small, of order a few. We can then write the $D$ dimensional Lagrangian as follows

$$
\mathcal{L}_{D} \sim \frac{N \Lambda^{D}}{\ell_{D}} \hat{\mathcal{L}}(\hat{\Phi}, \partial / \Lambda)
$$

where $\hat{\Phi}$ is a field whose kinetic term is not canonically normalized, $\Lambda$ is the cutoff of the theory, and all parameters in $\hat{\mathcal{L}}$ are dimensionless and $O(1)$.

\section{Gravity Lagrangian}

We can begin with the gravity Lagrangian of Eq. (A.1) and use the fact that the kinetic term has two derivatives to estimate the cutoff:

$$
\Lambda \sim\left(\frac{\ell_{5}}{N}\right)^{1 / 3} M_{5}=\left(\frac{3}{N}\right)^{1 / 3} 2 \pi M_{5}
$$

where $M_{5}$ is the five dimensional Planck mass. From this we see that there is a regime of the effective field theory before $5 D$ gravity becomes strongly coupled if $N$ is not too large. Next we estimate the natural value of the cosmological constant $\Lambda_{b}$

$$
\Lambda_{b} \sim \frac{N \Lambda^{5}}{\ell_{5}} \sim\left(\frac{\ell_{5}}{N}\right)^{2 / 3} M_{5}^{5}
$$

from which we can estimate the inverse of the AdS curvature $k$,

$$
k=\sqrt{\frac{-\Lambda_{b}}{24 M_{5}^{3}}} \sim \frac{1}{2 \sqrt{6}}\left(\frac{\ell_{5}}{N}\right)^{1 / 3} M_{5} \sim \frac{2}{N^{1 / 3}} M_{5} \sim \frac{\Lambda}{\sqrt{24}} .
$$

From this we see that the AdS curvature scale is only separated by a factor of a few from the cutoff $\Lambda$ and the higher dimensional Planck scale $M_{5}$. This implies that if the bulk cosmological constant is of order its natural value, only a handful of KK states are present below the cutoff.

The AdS/CFT correspondence assumes the hierarchy $M_{5} \gg M_{S} \gg k$. Here $M_{S}$ represents the string scale, the energy scale at which string excitations enter the picture. From the CFT perspective, this corresponds to requiring that $N_{c} \gg 1$ and $g^{2} N_{c} \gg 1$, where $N_{c}$ is the number of colors and $g$ the coupling constant in the dual gauge theory. The fact that $M_{5}$ and $k$ differ only by a factor of a few implies that we are not actually in the regime where $N_{c}$ and $g^{2} N_{c}$ are large. This implies that the results we obtain using our NDA estimates can only be taken as a very rough guide, since the correspondence is being pushed to the edge of its domain of validity.

We can also use NDA to estimate the natural values of the four dimensional cosmological constants, which in this case are the brane tensions:

$$
T_{h} \sim T_{v} \sim \frac{N \Lambda^{4}}{\ell_{4}} \sim \frac{\ell_{5}^{4 / 3}}{\ell_{4} N^{1 / 3}} M_{5}^{4} .
$$

These parameters are restricted to a four dimensional brane, so it is the four dimensional loop factor which goes into the estimate. 


\section{GW Field}

The next step is to estimate the values of the parameters in the potential of the GW field, $\Phi$. We begin with the bulk parameters defined in Eq. (2.13). In order to get from the Lagrangian in Eq. (C.2), to one with field that have canonical kinetic terms, we have to rescale $\Phi$ by $\sqrt{\ell_{5} / N \Lambda^{3}}$. Therefore, for a mass term $m^{2} \Phi^{2} / 2$, the natural value of the mass is given by

$$
m^{2} \sim \Lambda^{2} \sim 24 k^{2} .
$$

In order to generate a large hierarchy, the mass parameter is taken to lie significantly below its NDA value. The natural value of the cubic interaction is also easily obtained as

$$
\eta \sim \sqrt{\frac{\ell_{5} \Lambda}{N}} \sim 24^{1 / 4} \sqrt{\frac{\ell_{5} k}{N}} \sim 60 \sqrt{\frac{k}{N}} .
$$

We also want to compute the natural values of the parameters $\alpha$ and $v$. The visible brane potential for the GW field is given by

$$
V_{v}=\delta(\theta-\pi) 2 k^{5 / 2} \alpha \Phi .
$$

Using the NDA prescription, we estimate the size of this term to be

$$
\begin{gathered}
2 \alpha k^{5 / 2} \sim \frac{\sqrt{N \ell_{5}}}{\ell_{4}} \Lambda^{5 / 2} \sim \frac{\ell_{5}^{4 / 3}}{\ell_{4} N^{1 / 3}} M_{5}^{5 / 2} \\
\alpha \sim 2^{11 / 4} 3^{5 / 4} \frac{\sqrt{N \ell_{5}}}{\ell_{4}} \sim 3\left(\frac{54}{\pi^{2}}\right)^{1 / 4} \sqrt{N} \sim 5 \sqrt{N} .
\end{gathered}
$$

As discussed in section 2.1, we only require that $\Phi(\theta=0)=k^{3 / 2} v$, but leave the potential unspecified. One potential that can generate this boundary condition is

$$
V_{h}=\delta(\theta) \lambda\left(\Phi^{2}-k^{3} v^{2}\right)^{2},
$$

from which we estimate the natural value of $v$ as

$$
v \sim 24^{3 / 4}\left(\frac{N}{\ell_{5}}\right)^{1 / 2}=\frac{24^{1 / 4}}{\pi^{3 / 2}} \sqrt{N} \sim 0.4 \sqrt{N} .
$$

Using other possible potentials such as $\lambda\left(\Phi-k^{3 / 2} v\right)^{2}$ give the same estimate for $v$. In order to generate a hierarchy we take $v$ to lie below its NDA value.

\section{Radion Dynamics}

The radion parameter $\tau$ associated with the quartic is defined below Eq. (2.21) and can be estimated as follows:

$$
\tau=\frac{1}{k^{4}}\left(T_{v}-\frac{\Lambda_{b}}{k}-\frac{k^{4} \alpha^{2}}{4}\right) \sim 72 N\left(\frac{8}{\ell_{4}}-\frac{16 \sqrt{6}}{\ell_{5}}-\frac{\sqrt{6} \ell_{5}}{\ell_{4}^{2}}\right) \sim 5 N .
$$

The three contributions we show are roughly the same size. Since this is just an estimate, there are $O(1)$ coefficients on each term, so we assume there is no cancellation and that total size of $\tau$ is the size of each individual term. 
We have computed the mass of the radion in the regime where the OR Eq. (2.14) is dominated by $m^{2}$ and by $\eta$ in Eqs. (2.23) and (2.34) respectively:

$$
\frac{m_{\varphi}^{2}}{\left(k e^{-k \pi r_{c}}\right)^{2}}= \begin{cases}-\frac{\epsilon \tau}{6} \frac{k^{3}}{M_{5}^{3}} & \text { if } m^{2} \text { dominates } \\ \frac{\tau^{2} \xi}{6 w} \frac{k^{3}}{M_{5}^{3}} \sim 10 & \text { if } \eta \text { dominates. }\end{cases}
$$

These formulae require that the mass term be well below its natural value, so we only give an NDA estimate for the second case in which the radion mass does not depend on $\epsilon$. As the mass of the KK gravitons is typically $\sim 3$ times larger than $k e^{-k \pi r_{c}}$, we see that in the cubic case NDA gives us the expected result that the radion mass is roughly equal to the KK scale.

\section{Radion Coupling to SM}

Finally we estimate the coupling of the GW field to other Standard Model fields on the visible brane. These couplings take the form

$$
\left(1+\alpha_{\mathrm{int}} \Phi / k^{3 / 2}\right) \cdot \mathcal{O}_{\mathrm{SM}}
$$

We can estimate the size of $\alpha_{\text {int }}$ using NDA and assuming that the operator $\mathcal{O}_{\mathrm{SM}}$ has already been normalized, so we just need to rescale $\Phi$ to get a canonical kinetic term:

$$
\begin{gathered}
\alpha_{\text {int }} / k^{3 / 2} \sim \ell_{5}^{1 / 2} / N^{1 / 2} \Lambda^{3 / 2} \\
\alpha_{\text {int }} \sim \frac{1}{24^{3 / 4}} \sqrt{\frac{\ell_{5}}{N}} \sim \frac{1}{\sqrt{N}}\left(\frac{\pi^{6}}{24}\right)^{1 / 4} \sim \frac{2.5}{\sqrt{N}} .
\end{gathered}
$$

We will take these parameters to be their NDA sizes, but since they will always multiply $\Phi / k^{3 / 2}$, we will end up only working to first order in them.

\section{References}

[1] CMS Collaboration Collaboration, S. Chatrchyan et al., Observation of a new boson at a mass of $125 \mathrm{GeV}$ with the CMS experiment at the LHC, Phys.Lett. B716 (2012) 30-61, [arXiv: 1207.7235].

[2] ATLAS Collaboration Collaboration, G. Aad et al., Observation of a new particle in the search for the Standard Model Higgs boson with the ATLAS detector at the LHC, Phys.Lett. B716 (2012) 1-29, [arXiv: 1207.7214].

[3] L. Susskind, Dynamics of Spontaneous Symmetry Breaking in the Weinberg-Salam Theory, Phys.Rev. D20 (1979) 2619-2625.

[4] e. 't Hooft, Gerard, e. Itzykson, C., e. Jaffe, A., e. Lehmann, H., e. Mitter, P.K., et al., Recent Developments in Gauge Theories. Proceedings, Nato Advanced Study Institute, Cargese, France, August 26 - September 8, 1979, NATO Adv.Study Inst.Ser.B Phys. 59 (1980) pp.1-438.

[5] L. Randall and R. Sundrum, A Large mass hierarchy from a small extra dimension, Phys.Rev.Lett. 83 (1999) 3370-3373, [hep-ph/9905221]. 
[6] K. Agashe, A. Delgado, M. J. May, and R. Sundrum, RS1, custodial isospin and precision tests, JHEP 0308 (2003) 050, [hep-ph/0308036].

[7] K. Agashe, R. Contino, L. Da Rold, and A. Pomarol, A Custodial symmetry for Zb anti-b, Phys.Lett. B641 (2006) 62-66, [hep-ph/0605341].

[8] C. Csaki, C. Grojean, L. Pilo, and J. Terning, Towards a realistic model of Higgsless electroweak symmetry breaking, Phys.Rev.Lett. 92 (2004) 101802, [hep-ph/0308038].

[9] R. Contino, Y. Nomura, and A. Pomarol, Higgs as a holographic pseudoGoldstone boson, Nucl.Phys. B671 (2003) 148-174, [hep-ph/0306259].

[10] K. Agashe, R. Contino, and A. Pomarol, The Minimal composite Higgs model, Nucl.Phys. B719 (2005) 165-187, [hep-ph/0412089].

[11] Y. Grossman and M. Neubert, Neutrino masses and mixings in nonfactorizable geometry, Phys.Lett. B474 (2000) 361-371, [hep-ph/9912408].

[12] T. Gherghetta and A. Pomarol, Bulk fields and supersymmetry in a slice of AdS, Nucl.Phys. B586 (2000) 141-162, [hep-ph/0003129].

[13] K. Agashe, G. Perez, and A. Soni, Flavor structure of warped extra dimension models, Phys.Rev. D71 (2005) 016002, [hep-ph/0408134].

[14] K. Agashe and G. Servant, Warped unification, proton stability and dark matter, Phys.Rev.Lett. 93 (2004) 231805, [hep-ph/0403143].

[15] K. Agashe and G. Servant, Baryon number in warped GUTs: Model building and (dark matter related) phenomenology, JCAP 0502 (2005) 002, [hep-ph/0411254].

[16] A. D. Medina and E. Ponton, Warped Radion Dark Matter, JHEP 1109 (2011) 016, [arXiv:1104.4124].

[17] K. Agashe, A. Belyaev, T. Krupovnickas, G. Perez, and J. Virzi, LHC Signals from Warped Extra Dimensions, Phys.Rev. D77 (2008) 015003, [hep-ph/0612015].

[18] R. Contino, T. Kramer, M. Son, and R. Sundrum, Warped/composite phenomenology simplified, JHEP 0705 (2007) 074, [hep-ph/0612180].

[19] B. Lillie, L. Randall, and L.-T. Wang, The Bulk RS KK-gluon at the LHC, JHEP 0709 (2007) 074, [hep-ph/0701166].

[20] W. D. Goldberger and M. B. Wise, Modulus stabilization with bulk fields, Phys.Rev.Lett. 83 (1999) 4922-4925, [hep-ph/9907447].

[21] C. Csaki, M. Graesser, L. Randall, and J. Terning, Cosmology of brane models with radion stabilization, Phys.Rev. D62 (2000) 045015, [hep-ph/9911406].

[22] W. D. Goldberger and M. B. Wise, Phenomenology of a stabilized modulus, Phys.Lett. B475 (2000) 275-279, [hep-ph/9911457].

[23] G. F. Giudice, R. Rattazzi, and J. D. Wells, Graviscalars from higher dimensional metrics and curvature Higgs mixing, Nucl.Phys. B595 (2001) 250-276, [hep-ph/0002178].

[24] C. Csaki, M. L. Graesser, and G. D. Kribs, Radion dynamics and electroweak physics, Phys.Rev. D63 (2001) 065002, [hep-th/0008151].

[25] C. Csaki, J. Hubisz, and S. J. Lee, Radion phenomenology in realistic warped space models, Phys.Rev. D76 (2007) 125015, [arXiv:0705.3844]. 
[26] T. G. Rizzo, Radion couplings to bulk fields in the Randall-Sundrum model, JHEP 0206 (2002) 056, [hep-ph/0205242].

[27] J. M. Maldacena, The Large $N$ limit of superconformal field theories and supergravity, Adv. Theor.Math.Phys. 2 (1998) 231-252, [hep-th/9711200].

[28] E. Witten, Anti-de Sitter space and holography, Adv.Theor.Math.Phys. 2 (1998) 253-291, [hep-th/9802150].

[29] S. Gubser, I. R. Klebanov, and A. M. Polyakov, Gauge theory correlators from noncritical string theory, Phys.Lett. B428 (1998) 105-114, [hep-th/9802109].

[30] I. R. Klebanov and E. Witten, AdS / CFT correspondence and symmetry breaking, Nucl.Phys. B556 (1999) 89-114, [hep-th/9905104].

[31] N. Arkani-Hamed, M. Porrati, and L. Randall, Holography and phenomenology, JHEP 0108 (2001) 017, [hep-th/0012148].

[32] R. Rattazzi and A. Zaffaroni, Comments on the holographic picture of the Randall-Sundrum model, JHEP 0104 (2001) 021, [hep-th/0012248].

[33] A. Salam and J. Strathdee, Nonlinear realizations. 2. Conformal symmetry, Phys.Rev. 184 (1969) $1760-1768$.

[34] C. Isham, A. Salam, and J. Strathdee, Spontaneous breakdown of conformal symmetry, Phys.Lett. B31 (1970) 300-302.

[35] C. Isham, A. Salam, and J. Strathdee, Nonlinear realizations of space-time symmetries. Scalar and tensor gravity, Annals Phys. 62 (1971) 98-119.

[36] B. Zumino, Lectures on elementary particles and quantum field theory, in 1970 Brandeis Summer School (S. Deser, ed.). MIT Press, 1970.

[37] J. R. Ellis, Aspects of conformal symmetry and chirality, Nucl.Phys. B22 (1970) 478-492.

[38] J. R. Ellis, Phenomenological actions for spontaneously-broken conformal symmetry, Nucl.Phys. B26 (1971) 536-546.

[39] W. D. Goldberger, B. Grinstein, and W. Skiba, Distinguishing the Higgs boson from the dilaton at the Large Hadron Collider, Phys.Rev.Lett. 100 (2008) 111802, [arXiv:0708.1463].

[40] J. Fan, W. D. Goldberger, A. Ross, and W. Skiba, Standard Model couplings and collider signatures of a light scalar, Phys.Rev. D79 (2009) 035017, [arXiv:0803.2040].

[41] L. Vecchi, Phenomenology of a light scalar: the dilaton, Phys.Rev. D82 (2010) 076009, [arXiv: 1002.1721].

[42] R. Rattazzi, The Naturally Light Dilaton, . Planck 2010.

[43] Z. Chacko and R. K. Mishra, Effective Theory of a Light Dilaton, Phys.Rev. D87 (2013) 115006, [arXiv:1209.3022].

[44] B. Bellazzini, C. Csaki, J. Hubisz, J. Serra, and J. Terning, A Higgslike Dilaton, Eur.Phys.J. C73 (2013) 2333, [arXiv:1209.3299].

[45] T. Abe, R. Kitano, Y. Konishi, K.-y. Oda, J. Sato, et al., Minimal Dilaton Model, Phys.Rev. D86 (2012) 115016, [arXiv:1209.4544].

[46] F. Coradeschi, P. Lodone, D. Pappadopulo, R. Rattazzi, and L. Vitale, A naturally light dilaton, arXiv:1306.4601. 
[47] C. Charmousis, R. Gregory, and V. Rubakov, Wave function of the radion in a brane world, Phys.Rev. D62 (2000) 067505, [hep-th/9912160].

[48] M. A. Luty and R. Sundrum, Hierarchy stabilization in warped supersymmetry, Phys.Rev. D64 (2001) 065012, [hep-th/0012158].

[49] P. Breitenlohner and D. Z. Freedman, Stability in Gauged Extended Supergravity, Annals Phys. 144 (1982) 249.

[50] C. M. Bender and S. A. Orszag, Advanced Mathematical methods for Scientists and Engineers. McGraw-Hill Publishing Company, 1978.

[51] T. Konstandin, G. Nardini, and M. Quiros, Gravitational Backreaction Effects on the Holographic Phase Transition, Phys.Rev. D82 (2010) 083513, [arXiv:1007.1468].

[52] Y. Eshel, S. J. Lee, G. Perez, and Y. Soreq, Shining Flavor and Radion Phenomenology in Warped Extra Dimension, JHEP 1110 (2011) 015, [arXiv:1106.6218].

[53] H. Georgi and A. Pais, Calculability and Naturalness in Gauge Theories, Phys.Rev. D10 (1974) 539.

[54] H. Georgi and A. Pais, Vacuum Symmetry and the PseudoGoldstone Phenomenon, Phys.Rev. D12 (1975) 508.

[55] D. B. Kaplan and H. Georgi, SU(2) x U(1) Breaking by Vacuum Misalignment, Phys.Lett. B136 (1984) 183.

[56] D. B. Kaplan, H. Georgi, and S. Dimopoulos, Composite Higgs Scalars, Phys.Lett. B136 (1984) 187.

[57] H. Georgi and D. B. Kaplan, Composite Higgs and Custodial SU(2), Phys.Lett. B145 (1984) 216.

[58] C. Arzt, Reduced effective Lagrangians, Phys.Lett. B342 (1995) 189-195, [hep-ph/9304230].

[59] H. Georgi, On-shell effective field theory, Nucl.Phys. B361 (1991) 339-350.

[60] M. Porrati and A. Starinets, $R G$ fixed points in supergravity duals of 4-D field theory and asymptotically AdS spaces, Phys.Lett. B454 (1999) 77-83, [hep-th/9903085].

[61] V. Balasubramanian and P. Kraus, Space-time and the holographic renormalization group, Phys.Rev.Lett. 83 (1999) 3605-3608, [hep-th/9903190].

[62] H. L. Verlinde, Holography and compactification, Nucl.Phys. B580 (2000) 264-274, [hep-th/9906182].

[63] J. de Boer, E. P. Verlinde, and H. L. Verlinde, On the holographic renormalization group, JHEP 0008 (2000) 003, [hep-th/9912012].

[64] E. P. Verlinde and H. L. Verlinde, RG flow, gravity and the cosmological constant, JHEP 0005 (2000) 034, [hep-th/9912018].

[65] V. Balasubramanian, E. G. Gimon, and D. Minic, Consistency conditions for holographic duality, JHEP 0005 (2000) 014, [hep-th/0003147].

[66] S. de Haro, S. N. Solodukhin, and K. Skenderis, Holographic reconstruction of space-time and renormalization in the AdS / CFT correspondence, Commun.Math.Phys. 217 (2001) 595-622, [hep-th/0002230].

[67] A. Manohar and H. Georgi, Chiral Quarks and the Nonrelativistic Quark Model, Nucl.Phys. B234 (1984) 189. 
[68] H. Georgi and L. Randall, Flavor Conserving CP Violation in Invisible Axion Models, Nucl.Phys. B276 (1986) 241.

[69] Z. Chacko, M. A. Luty, and E. Ponton, Massive higher dimensional gauge fields as messengers of supersymmetry breaking, JHEP 0007 (2000) 036, [hep-ph/9909248]. 